Draft version March 30, 2021

Preprint typeset using $\mathrm{L}_{\mathrm{T}} \mathrm{EX}$ style emulateapj v. 08/22/09

\title{
VARIABILITY-BASED AGN SELECTION USING IMAGE SUBTRACTION IN THE SDSS AND LSST ERA
}

\author{
Yumi Choi $^{1}$, Robert R. Gibson ${ }^{1}$, Andrew C. Becker ${ }^{1}$, Željko Ivezić ${ }^{1}$, Andrew J. Connolly ${ }^{1}$, Chelsea L. \\ MACLEOD $^{2}$, John J. RuAn ${ }^{1}$, Scott F. Anderson ${ }^{1}$ \\ Draft version March 30, 2021
}

\begin{abstract}
With upcoming all sky surveys such as LSST poised to generate a deep digital movie of the optical sky, variability-based AGN selection will enable the construction of highly-complete catalogs with minimum contamination. In this study, we generate $g$-band difference images and construct light curves for QSO/AGN candidates listed in SDSS Stripe 82 public catalogs compiled from different methods, including spectroscopy, optical colors, variability, and X-ray detection. Image differencing excels at identifying variable sources embedded in complex or blended emission regions such as Type II AGNs and other low-luminosity AGNs that may be omitted from traditional photometric or spectroscopic catalogs. To separate QSOs/AGNs from other sources using our difference image light curves, we explore several light curve statistics and parameterize optical variability by the characteristic damping timescale $(\tau)$ and variability amplitude. By virtue of distinguishable variability parameters of AGNs, we are able to select them with high completeness of $93.4 \%$ and efficiency (i.e., purity) of $71.3 \%$. Based on optical variability, we also select highly variable blazar candidates, whose infrared colors are consistent with known blazars. One third of them are also radio detected. With the X-ray selected AGN candidates, we probe the optical variability of X-ray detected optically-extended sources using their difference image light curves for the first time. A combination of optical variability and X-ray detection enables us to select various types of host-dominated AGNs. Contrary to the AGN unification model prediction, two Type II AGN candidates (out of 6 ) show detectable variability on long-term timescales like typical Type I AGNs. This study will provide a baseline for future optical variability studies of extended sources.

Subject headings: galaxies: active — galaxies: nuclei — quasars: general — X-rays: general
\end{abstract}

\section{INTRODUCTION}

Most galaxies host supermassive black holes (SMBHs) at their central regions. It is believed that the SMBHs, especially when they are in an active or quasar mode, regulate the evolution of their host galaxies and their own growth through feedback processes. There is now strong evidence that active galactic nuclei (AGNs) shape not just their host galaxies (e.g., Di Matteo et al. 2005; Hopkins et al. 2007), but also the intracluster-medium in galaxy clusters (e.g., Begelman \& Celotti 2004), and even the ionization levels of the Universe on cosmological scales (e.g., Loeb \& Barkana 2001). Although the effects of AGN activity on larger-scales are well studied, a consensus has not been reached on the underlying physics that generates these effects. This is partly because we do not fully understand how AGN outflows evolve, and also because single-epoch studies only describe a snapshot of AGN activity.

AGNs seem to be powered by the accretion of matter onto SMBHs with complex hydrodynamic and magnetic processes, along with relativistic effects (e.g., Springel et al. 2005; McHardy 2010; Choi et al. 2012, and references therein). Unfortunately, we cannot directly observe the accretion disks in distant AGNs, as they are far too small to resolve. However, observations have shown that accretion disks surrounding the

\footnotetext{
Electronic address: ymchoi@astro.washington.edu

1 Department of Astronomy, University of Washington, Box 351580, Seattle, WA 98195, USA

2 Physics Department, U.S. Naval Academy, 572 Holloway Rd, Annapolis, MD 21402, USA
}

SMBHs often show a strong intrinsic variability in their optical/UV continuum. Optical variability seems to be a common trait of AGN activity and this variability can be measured out to high redshift in time-domain imaging surveys (e.g., Sesar et al. 2007). Although the physical mechanisms causing the variations in brightness are not yet fully understood, the intrinsic variability of AGN can provide keys to understanding its physical origin and the structure of their emission regions indirectly. Thus, one of the most promising approaches to understanding the physics and geometry of accretion processes is to examine the time variability of AGNs.

Studies of AGN variability across the electromagnetic spectrum (e.g., Simonetti et al. 1985; Hughes et al. 1992; Trevese et al. 1994; Kawaguchi et al. 1998; de Vries et al.|2005; Sesar et al.|2007; Bauer et al. 2009; Kelly et al. 2009; Kozłowski et al. 2010; Schmidt et al. 2010; MacLeod et al. 2011; Butler \& Bloom 2011; Kim et al. 2012) have already began paving the way for future time-domain surveys such as the Large Synoptic Survey Telescope (LSST; Ivezić et al. 2008), and are useful in current surveys such as Pan-STARRS (Kaiser et al. 2002) and the Palomar Transient Factory (PTF; Law et al. 2009). Optical variability will be an important means of identifying AGNs for upcoming imaging surveys since it is much more complete and efficient than the AGN selection based on optical colors alone (Butler \& Bloom 2011; MacLeod et al. 2011) and far less expensive than spectroscopic selection. Many efforts have been made to describe the distinctive optical variability of AGNs (characterized by non-periodicity and long timescales of variation) and its use for iden- 
tifying AGNs (e.g., de Vries et al. 2005). However, until recently, it was hard to provide simple selection criteria. Kelly et al. (2009) showed that optical AGN light curves are well described by a damped random walk (first order continuous autoregressive) model since the empirical power spectral distribution (PSD) of AGN light curves shares the same form $\operatorname{PSD}(f) \propto 1 / f^{2}$, where $\mathrm{f}$ is frequency, with the model. In this model, AGN light curves can be simply parameterized by a characteristic damping timescale and amplitude of variability. With a larger sample of light curves, Kozłowski et al. (2010) tested this model and concluded that it is robust and efficient for AGN selection. A recent study by MacLeod et al. (2011) showed that introduction of the damping timescale, $\tau$, into quasar selection can boost both completeness and efficiency. Furthermore, this photometric variability-based selection performs well even in the mid-z range $(2.2 \lesssim \mathrm{z} \leqslant 3.5)$, where optical color selection performs poorly (Schmidt et al. 2010).

AGN variability has also been studied in the X-ray, with recent studies characterizing typical variation amplitudes (e.g., Gibson \& Brandt 2012). The connection between optical/UV and X-ray emission provides important clues to the emission mechanism. Gibson et al. (2008) suggested the physical mechanism causing the global Baldwin effect (anti-correlation between the C IV emission line EW and the UV luminosity; Baldwin 1977) is also responsible for the X-ray emission. By incorporating information from the X-ray band, we will also be able to unveil any connections between the optical and $\mathrm{X}$-ray emitting regions.

In this work, we extend the findings of earlier photometric studies on quasar variability to a difference image analysis of Sloan Digital Sky Survey (SDSS; York et al. 2000) Stripe 82. Earlier studies were necessarily restricted to more luminous quasars since photometry is unreliable when the source cannot be modeled accurately due to blended galaxy emission. Our SDSS analysis opens up a new regime for difference image studies due to the size and coverage of Stripe 82 . While there are additional challenges to using difference images (described in Section 3.2), this method has the distinct advantage that it can be applied equally well to quasars and also AGNs surrounded by host-galaxy emission. Except for a few individual observations of nearby AGNs, previous studies generally have used ensembles of highly-luminous quasars that dramatically outshine their host galaxies. Of course, quasars represent only the brightest cases of AGN, a relatively small fraction of AGN overall. Many more AGNs have lower luminosities and are surrounded by significant galaxy emission. In order to truly study AGN demographics, influence, and evolution, we must expand our samples to this regime. Difference images can also be generated quickly in order to detect rapid brightness changes in near real-time. For these reasons, image differencing pipelines are integral to the data processing systems for imaging surveys such as the LSST and Pan-STARRS even if image differencing will be most useful for low redshift $(\mathrm{z}<0.5)$ objects only.

This paper is laid out as follows. In Section 2 we briefly summarize properties of 4 public catalogs of AGN candidates used in this study. Section 3 gives a description of observations and our data reduction of SDSS.
In Section 4, we describe the properties of the AGN candidate light curves in terms of the optical variability. We verify our methodology for characterizing difference image LCs, and then present the completeness and efficiency of our AGN selection for unresolved sources. We also examine relationships between their optical variability and X-ray properties. In Section [5 we discuss the results for optically-resolved, but X-ray-compact sources. Finally, we summarize and conclude in Section 6. Through out the paper, we adopt the cosmology $\mathrm{H}_{0}=70 \mathrm{~km} \mathrm{~s}^{-1} \mathrm{Mpc}^{-1}, \Omega_{m}=0.3$, and $\Omega_{\Lambda}=0.7$.

\section{CATALOGS OF CANDIDATE AGNS}

Many efforts have been made to compile large samples of quasars (extremely luminous AGNs) using a variety of approaches, including spectroscopy (e.g., Schneider et al. 2010), multi-band colors (e.g., Richards et al.|2009), optical variability (e.g., Sesar et al. 2007; Schmidt et al. 2010; MacLeod et al. 2011; Butler \& Bloom 2011), and X-ray detections (e.g., Brandt \& Hasinger 2005; Cardamone et al. 2008). Therefore, we utilize 4 existing catalogs of AGN candidates (for details, see Table 1 and the Appendix) compiled by each method, each with different degrees of purity, to determine what AGNs look like in a difference image survey. First, we use a catalog of spectroscopically identified quasars (hereafter "SpecQSO"), which serves as a test-bed for comparison between difference image and photometric LC analysis, and for examining the capability of our difference image techniques to identify AGNs. Second, we use a catalog of quasar candidates selected by optical colors (hereafter "PhotoQSO") to extend our tests to fainter sources and verify explicitly how well difference image techniques work in crowded fields, where multi-object spectroscopy suffers from fiber collision. Next, we use the Stripe 82 variable source catalog (hereafter "VarSrc") that contains many different types of variable objects, including quasars, to provide a sense of how well AGNs can be separated from other variables in terms of temporal variability. Finally, we use a catalog of X-ray detected AGN candidates (hereafter "XRaySrc") to examine Xray properties with optical variability. This enables us to explore, for the first time, the variability of opticallyextended (but compact in the X-ray) sources using difference image techniques. Before doing any analysis, we trim these catalogs to only select objects confined to the SDSS Stripe 82 (Section 3). Table 2 shows the fractions of sources which overlap between trimmed catalogs. To provide a sense of the physical characteristics of each catalog, we show the distribution of $g$ band magnitude in Figure 1 and the $g-r$ vs. $u-g$ color-color diagram (CCD) in Figure 2 Photometric information used in this study is from our Stripe 82 dataset (Section 3.1). The color-color space is divided into four characteristic regions (red dashed lines in Figure 2): Region I for lowredshift quasars, Region II for RR Lyrae stars, Region III for high-redshift quasars, and Region IV for stellar locus stars (Sesar et al. 2007). In the Appendix, we describe the origins and limitations of each catalog in more detail.

\section{OBSERVATIONS AND DATA REDUCTION}

The dataset for our difference image analysis comes from SDSS. The SDSS Stripe 82 imaging data cover 


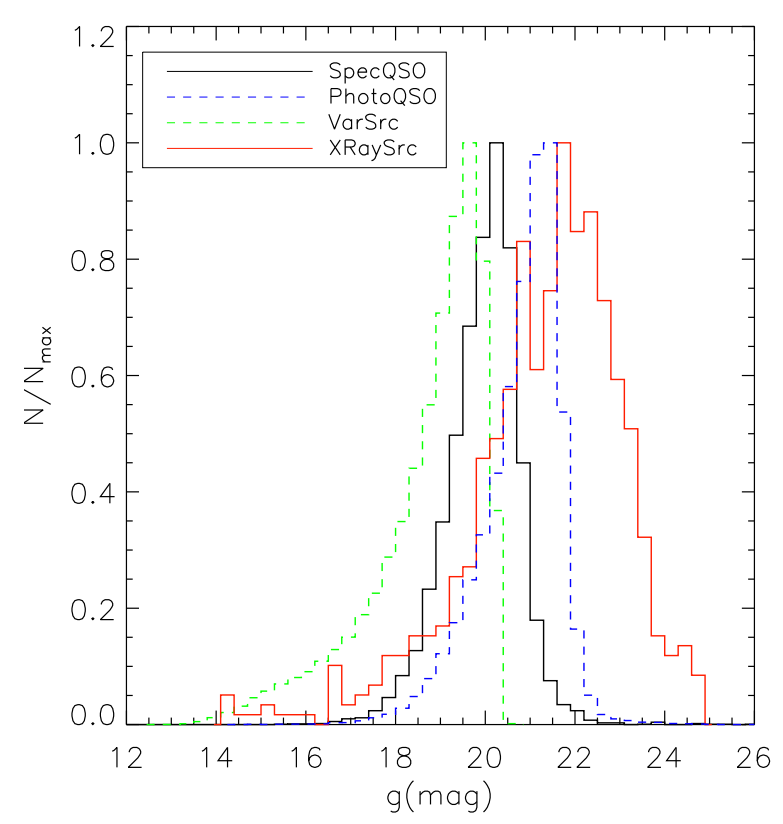

FIG. 1. - Normalized distribution of $g$-band PSF magnitude for each catalog (SpecQSO, PhotoQSO, VarSrc, XRaySrc). Total numbers of sources in each photometric catalog are listed in Table 1 The mean magnitudes are 19.93, 20.80, 18.72, and 21.33, for SpecQSO, PhotoQSO, VarSrc, and XRaySrc, respectively. From the XRaySrc catalog, here we present only point-like sources in the optical.

a large area of sky, $275 \operatorname{deg}^{2}\left(-50^{\circ}<\alpha_{J 2000.0}<60^{\circ}\right.$ and $\left.\left|\delta_{J 2000.0}\right|<1.25^{\circ}\right)$, with repeated observations over a decade in five bandpassess (ugriz; Fukugita et al. 1996). Its repeated observations and accurate photometry have enabled variability studies of RR Lyrae, supernovae, quasars, blazars, etc. (e.g., Sesar et al. 2007; Frieman et al. 2008; Sako et al. 2008; MacLeod et al. 2010). With $\sim 70$ observations per filter for $\sim 10$ years, supplemented by high-quality SDSS spectroscopy, Stripe 82 is a good test bed for a difference image study of AGN activity. In this study, we use both photometric (see Section 3.1) and difference image (see Section 3.2) data from Stripe $82 g$-band observations to explore AGN optical variability. We probe the $g$-band variability because shorter wavelengths generally show more AGN activity (e.g., Vanden Berk et al. 2004), while the SDSS $g$-band allows deeper images than the $u$ band. We will characterize photometric and difference image variability for the sources listed in 4 different public catalogs of AGN candidates.

\subsection{SDSS Photometry}

Ivezić et al. (2007) and Sesar et al. (2007, 2010) recalibrated the first and second phase of the SDSS (SDSS-I \& SDSS-II) multi-epoch imaging runs to improve photometric accuracy of data obtained in non-photometric conditions. These recalibrated data show typical photometric zero-point errors less than 0.02 mag. In Figure 3 . we show the mean number of photometric measurements and the mean maximum observation time interval as a function of RA for each catalogs. On average, this photometric data set reports PSF magnitudes for $\sim 50$ mea-
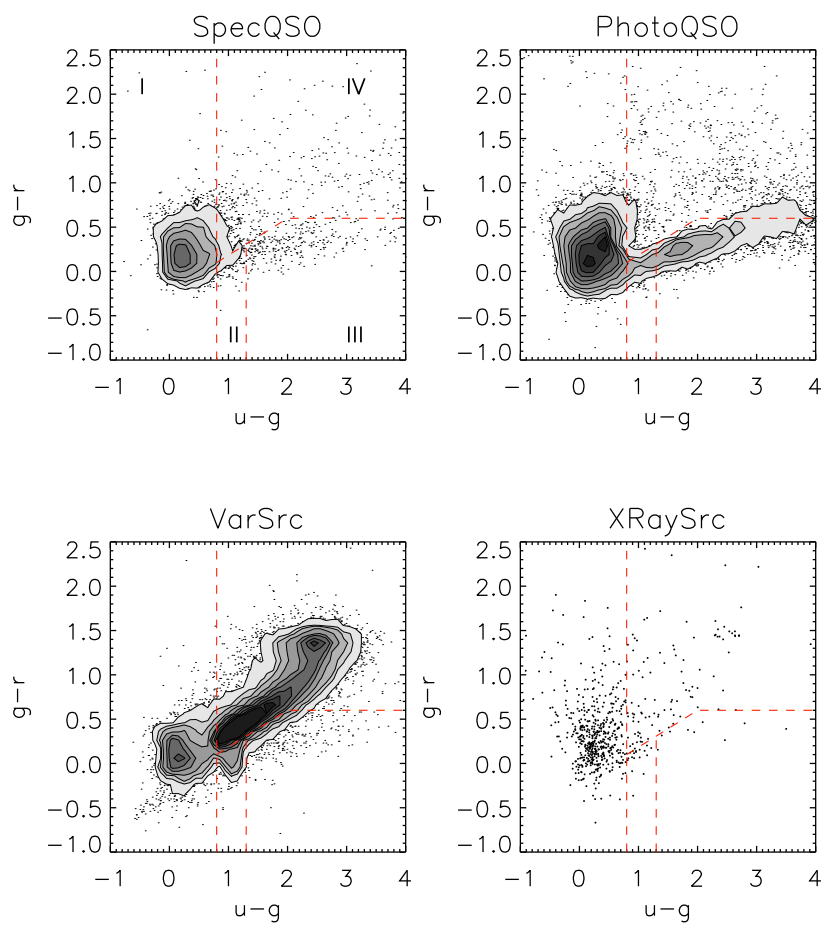

FIG. 2.- The $u-g$ vs. $g-r$ color-color diagram of each catalog. The contours show the number of data points at contour levels of $10,30,50,100,200,300,500,700$, and 900 . We do not show the contour lines for XRaySrc sample. The characteristic regions, which are defined in the color space (Sesar et al. 2007), are divided by the red dashed lines in each panel.

surements per unresolved object. For each source in the catalogs (Section 2), we construct $g$-band light curves (LCs) by matching all detections of each source across all runs in the recalibrated dataset with a $1^{\prime \prime}$ matching radius. These single-epoch catalog based LCs are referred to as photometric LCs to distinguish them from LCs constructed from difference images. For each photometric LC, we compute quantities such as the bias corrected sample standard deviation $\Sigma$, which is equivalent to the rms scatter in Sesar et al. (2007), reduced $\chi^{2}\left(\chi_{\nu}^{2}\right)$, and the structure function (SF) if the object is detected more than 4 times in the $g$-band (Section 4.1). Over $90 \%$ of all combined AGN candidates (SpecQSO, PhotoQSO, VarSrc, and XRaySrc) have at least 30 measurements in their photometric LCs.

\subsection{SDSS Difference Image}

Difference images are created by subtracting a deeper, coadded "template" image from a new observation (the "science image"). The template image is convolved with a spatially-varying kernel that matches it to the observing conditions of the science image. We refer the reader to details about the fundamentals of the image subtraction technique described in Alard \& Lupton (1998) and Alard (2000). Transient events and variable sources stand out in the difference images even when blended with extended sources such as host galaxies. This technique has been previously used to study quasars, supernovae, gamma-ray bursts, variable stars, microlensing events, and asteroids. The particular implementation we 

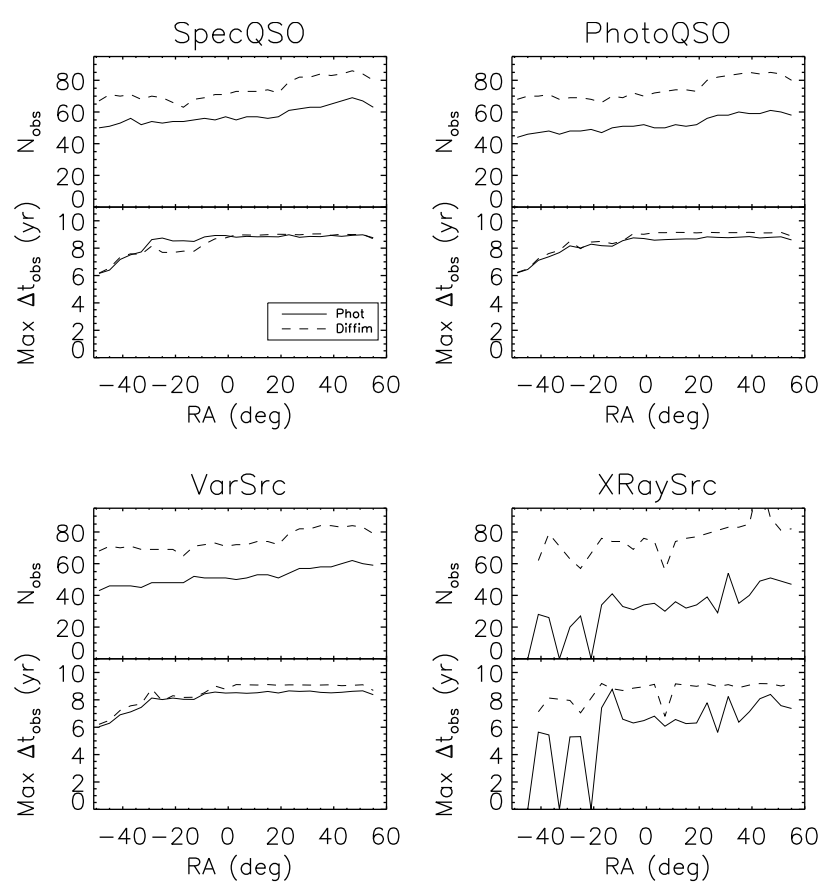

FIG. 3.- Number of detections (top in each panel) and the maximum $\Delta \mathrm{t}_{\text {obs }}$ (bottom in each panel) as a function of right ascension (RA). The average number of detections for SpecQSO, PhotoQSO, and VarSrc samples is about 70 epochs in difference image data, and is about 50 epochs in photometric data. However, there is no significant difference in their maximum $\Delta \mathrm{t}_{o b s}$ between difference image and photometric data, except for the XRaySrc. We note that many XRaySrc were not detected at many epochs or even any epochs in the optical photometric data. This is mainly because they are either faint or extended in the optical bands.

use in this study is developed for the LSST pipeline. ${ }^{3}$ First, we converted the SDSS imaging data (114,653 images across all runs in Stripe 82) into the LSST image format for use by the LSST software. A single image is 1489 pixels $\left(10^{\prime}\right)$ long and 2048 pixels wide $\left(13^{\prime}\right)$. We excluded 18 runs from the analysis that suffer from systematic issues such as improperly modeled gains for the CCD amplifiers and astrometric misalignments. We made use of data only in camera columns $2-5$ where the astrometric information in the image header was sufficient for image registration at the time when our difference images were generated (two outer columns have higher-order astrometric distortion).

We use template images (artificial run number 100006 for the south strip and 200006 for the north strip) of Stripe 82 coadded by Annis et al. (2011). They reach about 2 magnitudes deeper than a single run detection and have a median seeing of $1.1^{\prime \prime}$ in the $r$-band. These templates resulted from a median of the input data. Thus, the point spread function (PSF) of stars was seen to have a magnitude dependence, making PSF matching difficult and PSF photometry unreliable. For this reason, we use aperture photometry in our analysis below. Since any particular template image may not cover the exact same range of RA with its corresponding science image, we stitch together two adjacent template images when we generate a difference image (the Dec range varies much

\footnotetext{
${ }^{3}$ Available at http://dev.lsstcorp.org/cgit/LSST/DMS
}

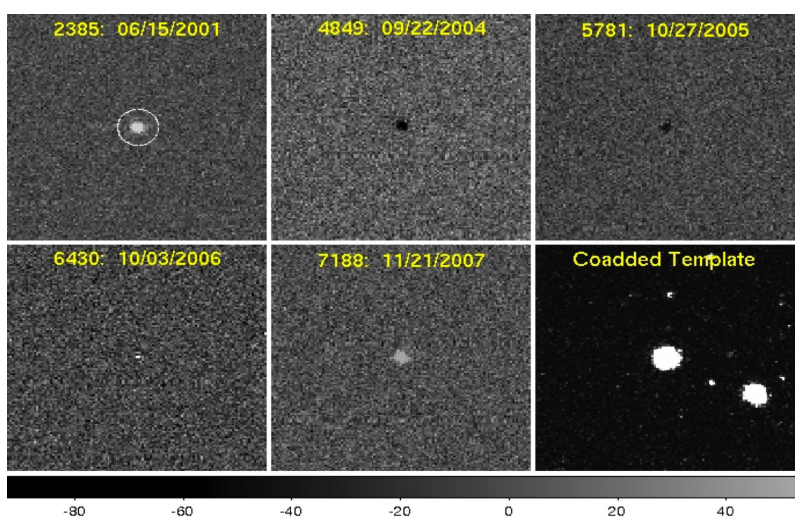

FIG. 4.- An example of spectroscopic quasar 316.00829 +0.38036 . Bottom right panel is the coadded template image around the quasar and the other panels are difference images of it at some randomly selected epochs. Run number and the date of observation are listed in each image.

less). Figure 4 shows an example of a spectroscopically confirmed quasar as seen in our difference images, including the coadded template image around the quasar. It is easy to see that residual fluxes from the object vary with time in the difference images.

We perform forced aperture photometry in each difference image to measure residual fluxes for each source listed in the 4 catalogs (Section 2). Forced aperture photometry is required in this case because summing over a sufficiently large aperture should cancel out any artificial spatial effects such as dipoles that may be produced, at the cost of $\mathrm{S} / \mathrm{N}$, since flux is still conserved even if the PSF matching shape is wrong. It also makes difference image LCs more complete since it is still possible to get a photometric data point from each epoch of observation even if the residual flux is negligible (but still non-zero) in a particular epoch difference image; correspondingly, the size of the flux measurement will be small relative to its error bar. By contrast, non-detections can be problematic in photometric LC analysis as pipelines may simply not report any epoch in which the source was not strongly detected, which could lead to significant differences in LC sampling and, accordingly, LC metrics.

The residual fluxes for each source are summed within three apertures having radii of $2^{\prime \prime}, 4^{\prime \prime}$, and $7^{\prime \prime}$ (the pixel scale of the SDSS is $\left.0.396^{\prime \prime}\right)$. These apertures are seeingcorrected to reflect variation of atmospheric seeing. No sources near the edge of an image are used in our study. If any pixel within the aperture has been masked, we flag the source for tracking purposes. Typically, a few masked pixels within the aperture do not affect the measurement of the residual flux. To make the sum of the residual fluxes for a given source from each difference image be comparable to one other, we divide this sum by the sum of the PSF matching kernels. This puts all the difference images on the photometric system of the common template image. The photometric uncertainty of each data point is estimated based on the variance plane of the science image that contains information on the total statistical uncertainty of a flux measurement, and then the measured uncertainty is also divided by the kernel sum. We empirically find that an aperture radius of $4^{\prime \prime}$ is large enough to enclose fluxes from most sources in our lists, while small enough to minimize noise and con- 


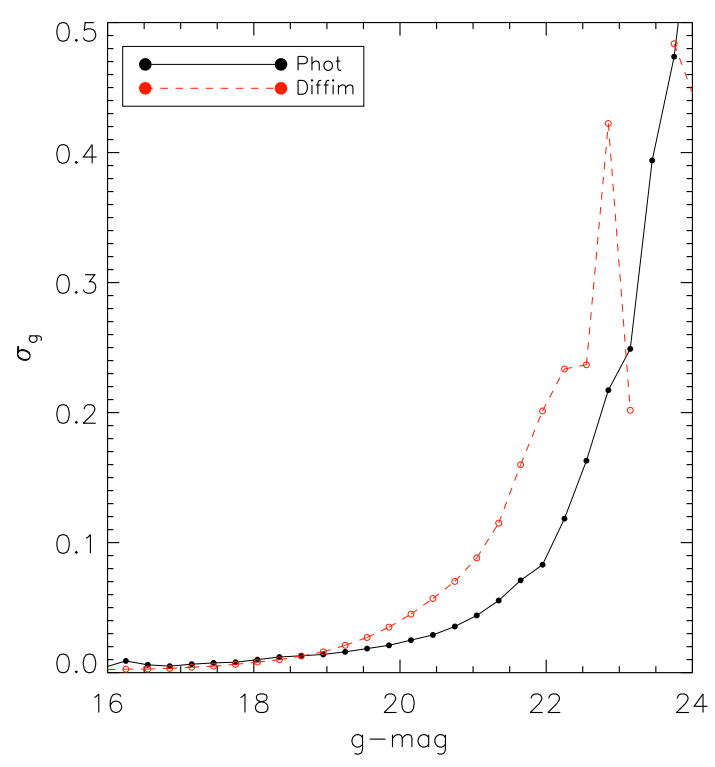

FIG. 5.- The median uncertainties of PSF photometry (black solid) and aperture photometry (red dashed) as a function of magnitude.

tamination. Therefore, difference image LCs constructed with the $4^{\prime \prime}$ aperture radius are used throughout the rest of this work.

In Figure [5, we compare the median uncertainties of PSF photometry and our aperture photometry on difference images as a function of $g$ band magnitude for the SpecQSO sample. The quality of aperture photometry is as good as PSF photometry for bright sources $(g \lesssim 19)$ and declines with magnitude rapider than PSF photometry $(10 \%$ worse at $g \simeq 22)$. Since the median dispersions of our difference image LCs as a function of magnitude are larger than the median uncertainties by a factor of 10 (2) at the bright (faint) end, we argue that our difference image LCs constructed from current template images (Annis et al. 2011) by performing aperture photometry are very reasonable for AGN variability study. Indeed, the difference image LCs reproduce their corresponding photometric LCs very well (Figure 6). Furthermore, there are, on average, $\sim 20$ more good data points in each difference image LC (dashed line in Figure 3) than its corresponding photometric LC (solid line in Figure 31). This is simply because the photometric recalibration was not generated for all runs in Stripe 82, while we perform forced aperture photometry on all difference images. However, there is no significant difference in the mean maximum observational time interval between the photometric and difference image data sets. This implies that, in most cases, missing runs in the photometric data sets are not from either the oldest or newest observations. We test the effect of missing data points on quantifying variability in Section 4.4.

In order to better understand the properties of difference image LCs, we also construct a control sample of LCs generated from 10,000 randomly-chosen positions on the sky. Since the randomly chosen spots are essentially empty spots on the difference images, they could represent either non-variable sources or more likely true empty spots on the sky. With the aid of the control

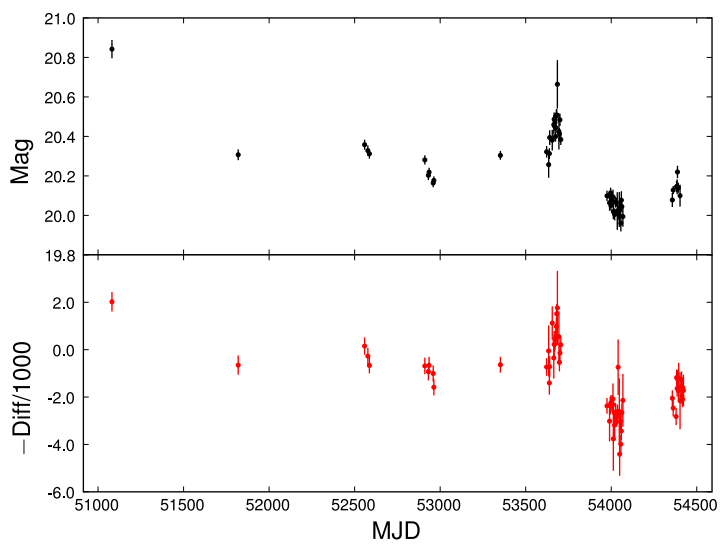

Fig. 6.- An example comparison between a photometric LC (top) and difference image LC (bottom) for an object with $g \simeq 20.3$. Although the photometric LC is in linear scale and the difference image LC is in log scale, they show the same fluctuation pattern. The difference image LC has relatively larger measurement errors than the photometric LC due to using aperture photometry on the difference images. Note that photometric errors do not strictly correlated with the source brightness since the supernova survey took data in a variety of observing conditions.

sample, we are able to provide a quantitative dividing line between non-variable and variable sources, or even between AGNs and other variable sources (Section 4.5).

\section{ANALYSIS OF UNRESOLVED SOURCES}

In this section, we describe our metrics and methodology to characterize LCs of all AGN candidates and present results for optically-unresolved sources.

\subsection{Light Curve Metrics}

To characterize AGN variability both in photometric and difference image LCs, we employ several LC statistics such as the $\Sigma, \chi_{\nu}^{2}$, and the first-order SF defined as

$$
\begin{aligned}
\Sigma & =\sqrt{\frac{1}{N-1} \sum_{i=1}^{N}\left(x_{i}-\bar{x}\right)^{2}}, \\
\chi_{\nu}^{2} & =\frac{1}{N-1} \sum_{i=1}^{N} \frac{\left(x_{i}-\bar{x}\right)^{2}}{\xi_{i}^{2}}, \\
S F(\Delta t) & =\sqrt{<\left(x_{t}-x_{t+\Delta t}\right)^{2}>},
\end{aligned}
$$

respectively. Here, $\mathrm{N}$ is the number of detections, $x_{i}$ $\left(x_{j}\right)$ is magnitude or flux at time $t_{i}\left(t_{j}\right), \xi_{i}$ is the error on the measurement, $\bar{x}$ is the error-weighted mean measurement value, $\bar{x}=\frac{\sum_{i=1}^{N} x_{i} / \xi_{i}^{2}}{\sum_{i=1}^{N} 1 / \xi_{i}^{2}}$, and $\Delta t$ is the time lag in the observer's frame. Using the time lag in the quasar rest frame would require either spectroscopic observations or some estimation of photometric redshift. If object redshift is not known and one is only interested in selecting quasars from other sources based on their optical variability, the use of the time lag in the observer's frame is sufficient (Schmidt et al. 2010; MacLeod et al. 2012). The SF analysis has been widely used to quantify AGN variability and constrain its variability models from observations with minimal assumptions, even when the data contain irregular tempo- 

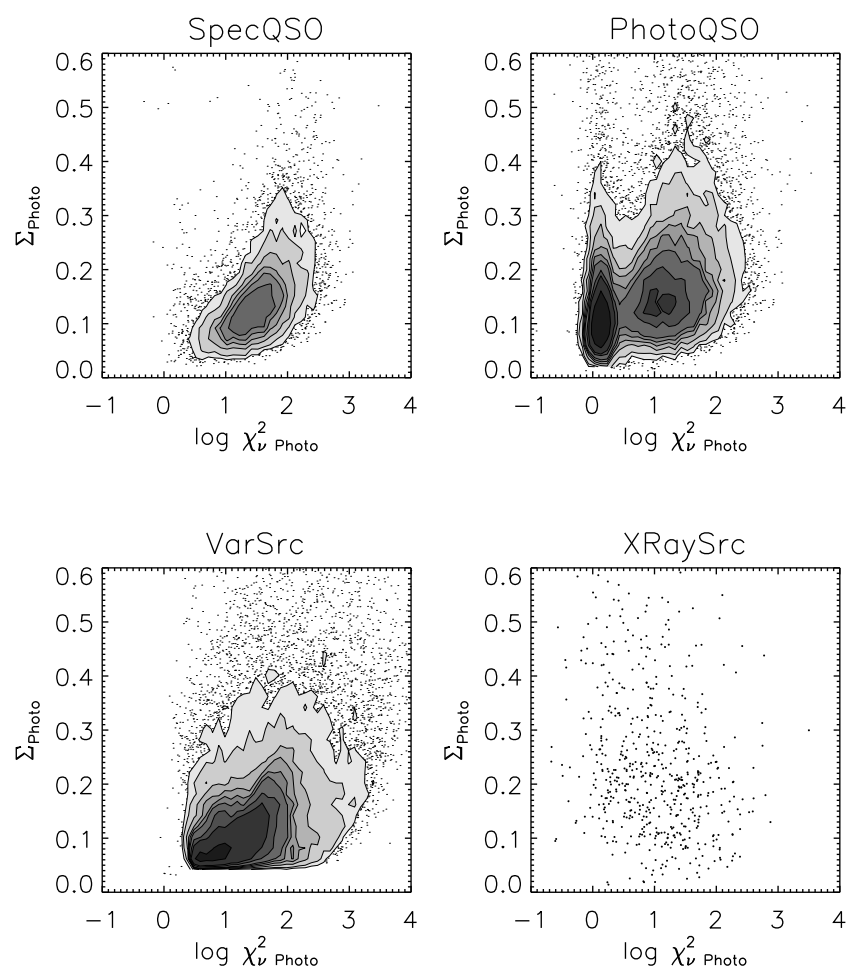

FIG. 7. $-\Sigma$ vs. $\chi_{\nu}^{2}$ from optical photometric LCs. The contours show the number of data points at contour levels of 10,20,40,60, $80,100,150,200$, and 400 .

ral gaps (e.g. Simonetti et al. 1985; Hughes et al. 1992; Kawaguchi et al. 1998; de Vries et al. 2005).

We first examine the distributions of $\Sigma$ and $\chi_{\nu}^{2}$ measured from both photometric and difference image LCs, and then present the SF analysis in the following section.

In Figure 7 , we show the $\Sigma$ and $\chi_{\nu}^{2}$ values computed from photometric LCs. Since the SDSS photometric error distribution is well described by a Gaussian distribution (Ivezić et al. 2007), large $\chi_{\nu}^{2}$ values can be used to select intrinsically variable sources. By placing selection cuts on the $\chi_{\nu}^{2}$ and the rms scatter ( $\Sigma$ in this work), Sesar et al. (2007) were able to reduce the fraction of false positives at the faint end where non-variable sources with large photometric uncertainties could mimic intrinsically variable sources with large values of rms scatter. Their selection cuts for variable sources were $\Sigma \geq 0.05$ and $\chi_{\nu}^{2} \geq 3$ in both the $g$ - and $r$-bands. About $97.9 \%$ of the SpecQSO sample (top left panel) pass the $g$-band selection cuts, confirming that a large fraction of quasars exhibit intrinsic variability in the optical. We note that SpecQSO objects with larger $\chi_{\nu}^{2}$ generally tend to have larger $\Sigma$, and only a few objects have $\log \left(\chi_{\nu}^{2}\right) \gtrsim 2.7$. This sharp edge is set by the bright sources in the catalog.

On the other hand, there are many objects in the PhotoQSO sample showing $\chi_{\nu}^{2}<3$ (about 41\%) and there is a clear bimodality in the $\log \left(\chi_{\nu}^{2}\right)$ distribution around $\chi_{\nu}^{2}=3$. This is not surprising, as these objects are either possible contaminants by non-variables or faint variables, and so their observed $\Sigma$ are likely dominated by Gaussian photometric noise. Since larger photometric scatter for faint non-variables can produce large $\Sigma$ values, we conclude that $\Sigma$, which is not normalized by uncertainty, by itself can not provide a good criterion for selecting intrinsically variable objects. The same trend between $\Sigma$ and $\chi_{\nu}^{2}$, seen in the SpecQSO sample, should occur for brighter sources only ( $g$ less than the mean magnitude of the PhotoQSO catalog), since fainter sources in the PhotoQSO catalog make the $\Sigma$ distribution broader.

Like the SpecQSO sample, most VarSrc objects $(\sim 96.4 \%)$ have $\Sigma \geq 0.05$ and $\chi_{\nu}^{2} \geq 3$. A few VarSrc objects, however, do not pass the current selection cuts while they all passed the cuts in Sesar et al. (2007). This small discrepancy is merely due to a difference in the definition of the mean; we use an error-weighted mean while they used an unweighted mean. Although most objects in this sample show significant level of variability, the correlation between their $\Sigma$ and $\chi_{\nu}^{2}$ is apparently weaker than the SpecQSO sample, but still present. This may be because the VarSrc catalog includes several different classes of variable sources besides quasars. Existence of very bright sources, mostly stars, in the catalog allows large $\chi_{\nu}^{2}$ values $\left(\gtrsim 10^{2.7}\right)$. Over $81 \%$ of sources with $\chi_{\nu}^{2} \gtrsim 10^{2.7}$ are brighter than $i=17$.

Based on the $\Sigma$ and $\chi_{\nu}^{2}$ cuts, about $73.7 \%$ of the XRaySrc objects having photometric LCs are expected to be intrinsically variable. However, even if we select variable candidates from the XRaySrc catalog by utilizing both $\Sigma$ and $\chi_{\nu}^{2}$ together, we are unable to tell whether these sources are AGNs or other X-ray luminous variables solely based on these cuts. Therefore, a SF analysis of their optical LCs is required to identify AGNs from other types of (non-)variables. Furthermore, the positive correlation between these two values, seen in the SpecQSO sample at $\chi_{\nu}^{2} \geq 3$, is not found in the XRaySrc sample. This is, again, likely due to both faint objects with large uncertainties and contamination by other sources (e.g., late-type flaring stars) other than AGNs/QSOs in the catalog.

Figure 8 shows the distributions of the $\Sigma$ and $\chi_{\nu}^{2}$ values for the same types of objects as in Figure 7 but derived from difference image LCs. The lack of clear bifurcation in the $\chi_{\nu}^{2}$ distribution for the PhotoQSO sample, which is seen in Figure [7, is due to the relatively large uncertainties on the difference image aperture photometry. We compare the distributions of $\chi_{\nu}^{2}$ measured from photometric and difference image LCs in Figure 9. In general, the distribution of $\chi_{\nu}^{2}$ from difference image LCs is shifted to smaller $\chi_{\nu}^{2}$ values compared to the photometric cases. The main reason for this is also the lower $\mathrm{S} / \mathrm{N}$ in flux measurements from difference image aperture photometry. We discuss this further in Section 4.4.

\subsection{Structure Function For the Damped Random Walk Model}

Stochastic optical variability is believed to be intrinsic to quasars. The amplitude of variability is greater on longer timescales, from months to years. Although several physical mechanisms, including accretion disk instabilities, starbursts, and microlensing, have been suggested, a physical origin for the quasar variability still remains an open question. Kelly et al. (2009) adopted a first order continuous autoregressive process, the damped random walk (DRW), to statistically model the observed light curves of quasars, and it has been shown that the DRW model can successfully describe the observed 

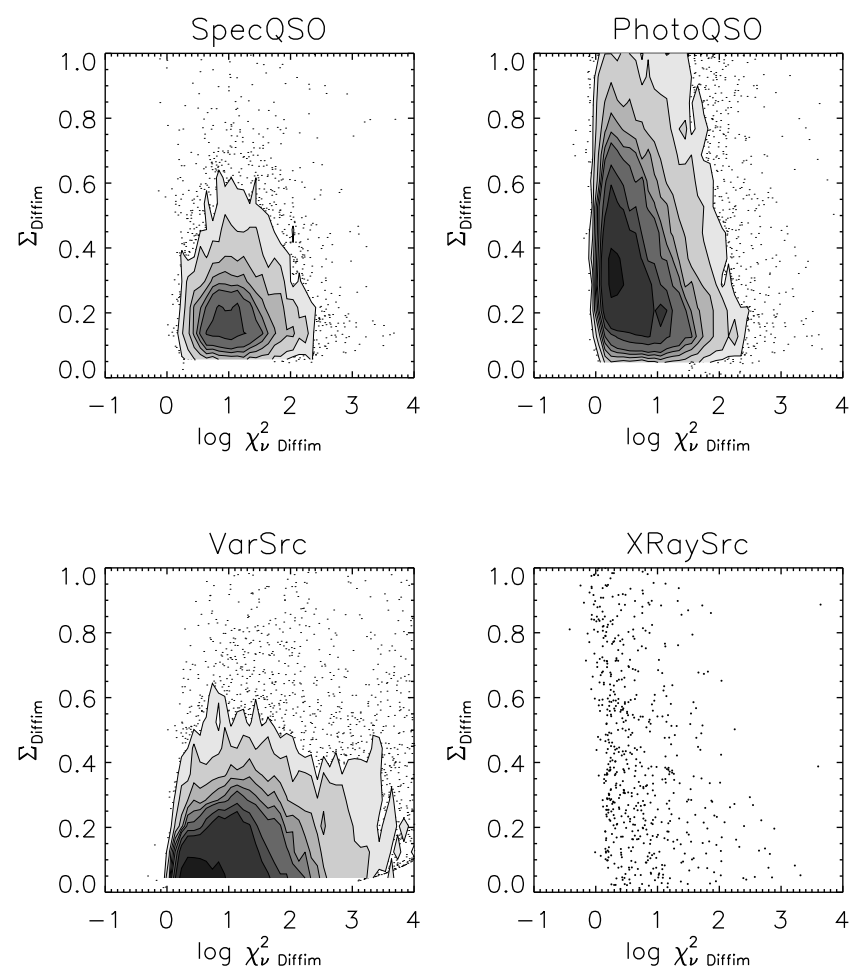

FIG. 8. - The same as Figure 6 but values obtained from difference image LCs.


FIG. 9.- Distributions of $\chi_{\nu}^{2}$ for sources having both photometric and difference image LCs. Black line is for photometric and magenta line is for difference image LCs. The dashed line shows $\chi_{\nu}^{2}=3$. Differences between distributions of $\chi_{\nu}^{2}$ mostly result from low $\mathrm{S} / \mathrm{N}$ in difference image LCs.
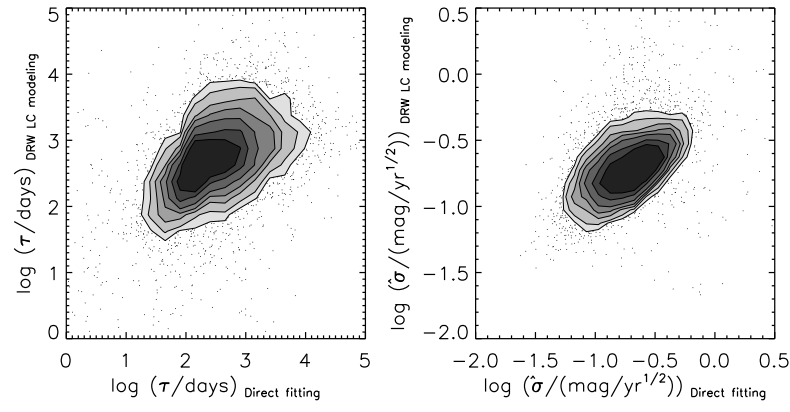

FIG. 10.- Comparison of the $\tau$ (left panel) and $\hat{\sigma}$ (right panel) derived from the two methods (direct fitting vs. DRW LC modeling) for the SpecQSO photometric LCs.

light curves of individual quasars using large samples (Kozłowski et al. 2010; MacLeod et al. 2010). The DRW model is a stochastic process with an exponential covariance

$$
S_{i j}\left(\Delta t_{i j}\right) \equiv \frac{1}{2} \hat{\sigma}^{2} \tau \exp \left(-\left|\Delta t_{i j}\right| / \tau\right),
$$

where $\hat{\sigma}$ controls the standard deviation of short-term variation, and $\tau$ is a characteristic damping timescale in days. To express the long-term variability of quasars characterized by the DRW as a function of the time lag, MacLeod et al. (2010) used the first-order SF, defined as

$$
S F(\Delta t) \equiv S F_{\infty}\left(1-e^{-|\Delta t| / \tau}\right)^{1 / 2} .
$$

The $\mathrm{SF}$ reaches an asymptotic value $\mathrm{SF}_{\infty}=\hat{\sigma} \sqrt{\tau}$ at large time lags, $|\Delta t| \gg \tau$. At small $|\Delta t|, \operatorname{SF}(\Delta t \ll$ $\tau)=\hat{\sigma} \sqrt{|\Delta t|}$. These SF parameters have been found to be closely correlated with physical parameters of quasars such as black hole mass and luminosity (e.g., MacLeod et al. 2010, 2012).

In this work, we use two different methods to obtain the best-fit SF parameters for individual objects:(i) estimating $\tau$ and $\mathrm{SF}_{\infty}$ by directly fitting the exponential form of SF (Equation 5) to observed SFs (Equation 3) using a least square fit, and (ii) estimating the $\tau$ and $\hat{\sigma}$ by modeling individual light curves using the method of Kozłowski et al. (2010). Their method to estimate these parameters is based on the approach of Press et al. (1992) (see the Appendix of Kozłowski et al. 2010). Note that these two methods constrain the amplitude of variability on differing timescales: the former method is more sensitive to long term parameter $\left(\mathrm{SF}_{\infty}\right)$, while the latter method constrains the short term amplitude $(\hat{\sigma})$ better. This, and the intrinsic differences in the fitting procedure between these two algorithms result in a systematic difference between their best-fit SF parameters (see Figure 10), although the results are in agreement in general. Since the former gives more weight to the larger time lag regime where most data points reside in observed SFs, it naturally returns smaller $\mathrm{SF}_{\infty}$ and shorter $\tau$ values compared to those derived from the latter. One can easily see this effect in examples of observed SFs for some SpecQSO objects in Figure 11.

To be able to compare difference image results with the previous analyses, which were conducted in magnitude, we test if the same characteristic timescales are obtained for a given LC analyzed both in magnitudes and in fluxes 


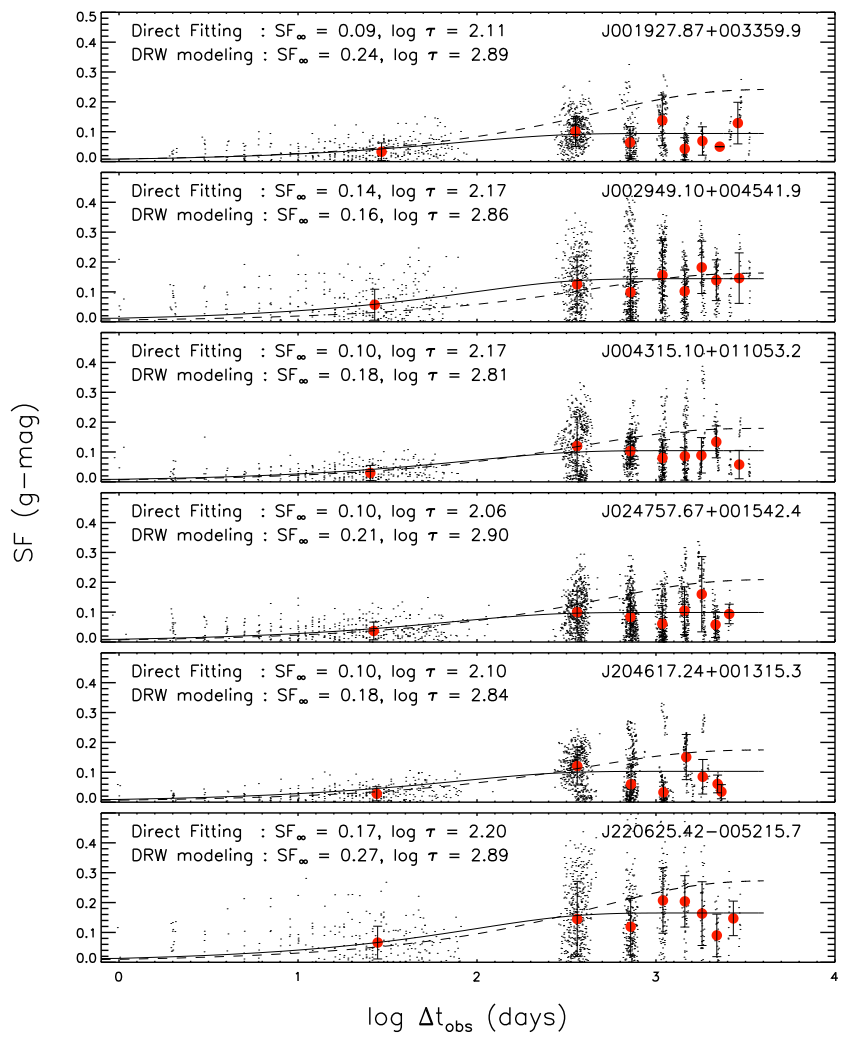

FIG. 11. - Examples of empirical SFs for some SpecQSO objects Black dots represent the observed SF and red dots are median SF values in each time lag bin. The solid (dashed) line shows the best-fit obtained by the direct fitting method (DRW LC modeling). Note that we are simultaneously fitting the entire time series instead of the binned values.

by a given LC fitting methodology. The test results show that the both methods are barely sensitive to the linear versus log scales. Further details on the test are described in the Appendix.

\subsubsection{Outliers and Uncertainty Estimation}

Dealing with LC outliers in variability metrics always has been problematic because they are not trivial to identify. One simple way to omit outliers from a light curve is to use deviation from the mean brightness. For example, one can define the most outlying point as an outlier or exclude data points which are more than $3 \sigma$ from the LC mean. However, it is often difficult to tell whether they are genuine outliers, especially for variable sources. Visual inspection can help in removing outliers from an individual LC, but this will be increasingly difficult for very large sample sizes. It also can cause human bias.

To check for the outlier sensitivity (i.e., LC robustness) and estimate the standard errors of the best fit parameters, we perform jackknife resampling for both methods rather than rejecting outliers based on arbitrary criteria. For each source, we repeat the fitting process every time after each data point in the $\mathrm{LC}$ is removed. When there are $\mathrm{N}$ data points in a $\mathrm{LC}$, we can have $\mathrm{N}$ best-fits for each measured parameter, and then compute the rms and the median of the SF parameters from $\mathrm{N}$ best-fits. In some cases, we note that both methods are sensitive to adding or omitting even just one data point in a LC regardless of the total number of measurements. In addi- tion to cases where LCs have actual outliers, we also find some cases where the SF parameter estimation largely depends on just one data point due to insufficient light curve lengths. Therefore, to only use well constrained (i.e., robust) LCs, we exclude sources (32\% of the SpecQSO) if they have $\tau$ of about zero (the "noise" limit) or infinity (the "run-away" limit) at least once among $\mathrm{N}$ best-fits of $\tau$, or have a rms in $\log \tau \geq 0.15$. Corresponding to these conservative robustness cuts, the fractions of robust LCs in the PhotoQSO, VarSrc, and XRaySrc catalogs are $46 \%, 35 \%$, and $34 \%$, respectively. The fraction seems to decrease as either the contamination by other sources increases or the number of faint sources increases in the catalog.

In Figure [12, we compare $\log \tau$ values for wellconstrained photometric LCs of the SpecQSO sample, estimated from the two fitting methods. They are well related to each other, with a slope of about unity. We also find that most excluded sources show a broader correlation and tend to have either long characteristic damping timescales, $\log \tau>3$, which is close to the maximum time lag of Stripe 82 data, or very short damping timescales, $\log \tau<0$. The non-zero intercept likely results from fundamental differences in the fitting procedure between the two methods mentioned above, which can lead to systematic differences in estimated $\tau$. Figure 12 also shows the comparison of best-fit $\hat{\sigma}$ for well-constrained LCs. There is good agreement between results from the two methods.

In this section, we have shown that the simple direct fitting method is sufficient enough to estimate SF parameters. Systematic differences from the DRW LC modeling can be taken into account when identifying AGNs in the SF parameter space (Section 4.5). We have also shown that the direct fitting method is insensitive to the scales of a LC (flux vs. magnitude). Therefore, we will focus on the best-fit SF parameters estimated by the simple direct fitting method, which is computationally less intensive, in our further analysis.

\subsection{Comparison of Photometric and Difference Image Light Curves}

Insensitivity of the direct fitting method to the LC scales allows SF analysis on difference image LCs, which are solely in flux, and a direct comparison of photometric LCs to common-epoch difference image LCs in terms of the best-fit $\tau$ values to see if there are any differences. We then compare photometric LCs to all-epoch difference image LCs to explore the effect of number of photometric measurements on the SF parameter estimation. Again, all LCs used in this section are from sources in the SpecQSO catalog.

First, we construct a set of difference image LCs having exactly the same-epoch data points as their counterpart photometric LCs. The common epoch requirement reduces the sample size. However, this strict condition ensures that we can detect any possible differences between the results of the SF analysis from photometric and difference image LCs, mainly caused by their different photometry (PSF vs. aperture), and thus, different $\mathrm{S} / \mathrm{N}$. Using the direct fitting method, we estimate the SF parameters for the set of common-epoch difference image LCs. For the comparison of $\log \tau$ values between photometric and difference image LCs, we have the slope of $1.006 \pm 0.167$ and the $\mathrm{y}$-intercept of $0.271 \pm 0.339$ (larger 

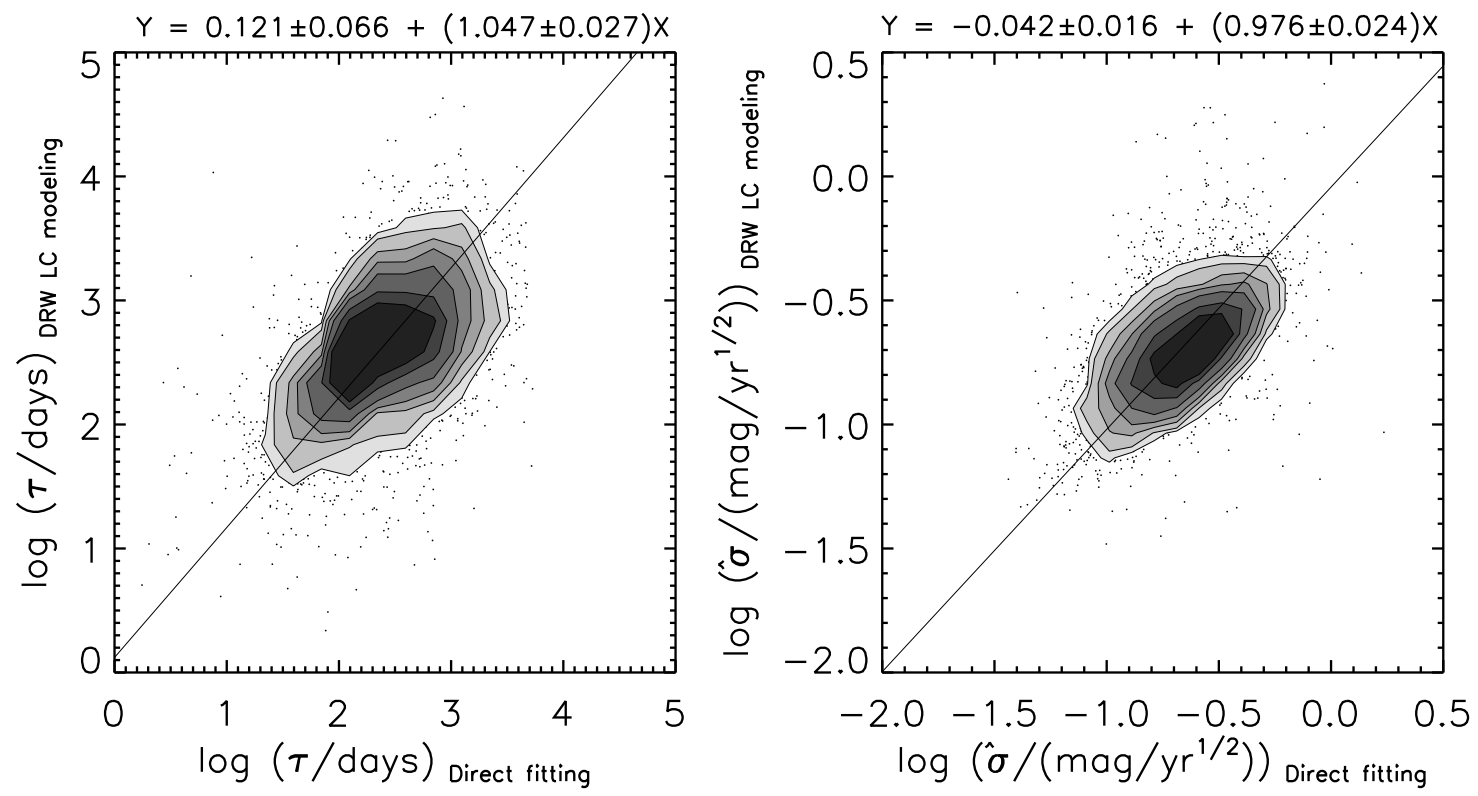

FIG. 12.- Left panel: Comparison of the $\tau$ for robust photometric LCs ( $\log \tau<5$ and $\operatorname{rms}$ of $\log \tau \leq 0.15)$ of the SpecQSO sample measured by the two different methods. The best-fit linear regression lines is overplotted. Right panel: Same as in the left panel, but comparison of the $\hat{\sigma}$.

$\log \tau$ for photometric LCs). Here, the large uncertainties are likely due to the small sample size. A non-zero intercept indicates that the lower $\mathrm{S} / \mathrm{N}$ of difference image LCs, which merely resulted from aperture photometry, can affect the estimation of $\tau$ in a way that systematically decreases it. This implies that our current difference image LCs might be less sensitive to low levels of variability, which is usually linked to short damping timescales, than photometric LCs. We repeat the same test with the DRW LC modeling and find the same trend. We expect that this will be improved by doing PSF photometry on the difference images generated from more precise PSF-matched template images in future works (e.g., https://dev.lsstcorp.org/trac/wiki/DC/Winter2013).

However, ideally we should be able to constrain variability of AGNs better with a difference image LC since it has, on average, $\sim 20$ more data points than a photometric LC. To confirm this, we use the all-epoch difference image LCs of the same objects in the common-epoch LC set. Firstly, with all-epoch difference image LCs, we can estimate the SF parameters for about $14 \%$ more objects that were rejected in the common-epoch LC set due to lack of number of measurements $(\mathrm{N}<4)$. Secondly, more objects (about $6 \%$ of the sample) pass the LC robustness cuts while they were described as unconstrained with the common-epoch LCs (i.e., the $\operatorname{rms}$ of $\log \tau \geq 0.15$, the run-away limit, or the noise limit). Thus, we conclude that more data points in a LC can definitely help in constraining AGN variability by increasing number of objects passing the initial $\mathrm{N}_{\text {obs }}$ cut and improving the LC robustness. We expect that this somewhat compensates for the disadvantage of the lower $\mathrm{S} / \mathrm{N}$ in difference image LCs for now, and eventually leads to significant improvements in the context of AGN identification in difference images with PSF photometry in the future. We will dis- cuss advantages and limitations of difference image LCs in more detail in the following section.

\subsection{Advantages and Limitations of Difference Image Light Curves}

Variability-based AGN selection methods have been limited to mostly point-like sources in the optical. This is because light contamination by extended host galaxies may dilute the change in brightness arising from relatively weak accretion activity, and the lower variability amplitudes result in difficulties in identifying them as AGNs. Thus image differencing works best in selecting AGNs embedded in extended host galaxies or lowerluminosity AGNs since it does not need to assume or fit a source model, and only the variable nuclei will stand out in difference images. Difference image LCs that are free of host galaxy contamination enable us to apply the variability-based selection to host-dominant AGNs as well. We will explore the variability properties of X-ray detected optically-extended sources, which are candidate faint AGNs, in Section 5 ,

As described in Section 3.2 a difference image LC contains, on average, about 20 more detections compared to its counterpart photometric LC. Figure 13 shows some examples of difference image LCs that illustrate the effect of more detections on the estimation of $\log \tau$. Even if the maximum time lag of observation is similar, missing data points between measurements makes a significant difference in the LC cadence (top panel of Figure 13), leading to the different SF parameters. The length of LCs is another important factor in determining variability properties. MacLeod et al. (2011) tested the impact of LC length on the best-fit distribution of the SF parameters and found a significant bias in $\log \tau$ and $\mathrm{SF}_{\infty}$ when estimated from LCs with a length sig- 

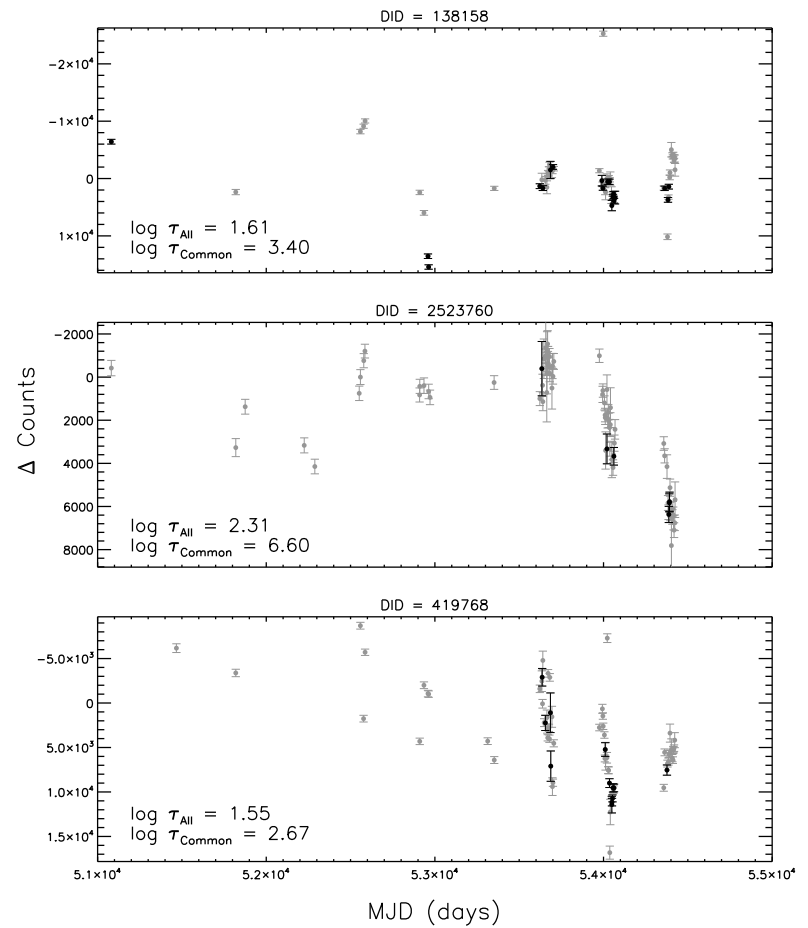

FIG. 13. - Examples of LCs to show the effect of more data points in a LC on estimating the SF parameters, especially $\tau$. Gray dots are for difference image LC data points and black dots are for the common-epoch data points. Top: SDSS J004032.10-001350.8 Middle: SDSS J005149.24-000133.5 Bottom: SDSS J230946.14+000048.8

nificantly shorter than 10 years. They showed $\tau$ can be easily overestimated from short LCs. Indeed, we see the impact of the LC length on $\tau$ in the middle and bottom panels of Figure 13. The common-epoch data cover only a small fraction of the entire temporal baseline, and returns very different $\tau$ values (larger than those measured from all-epoch data, even returning a run-away timescale for some cases). These results suggest that using LCs with more measurements (i.e., difference image LCs in this study) to characterize AGN variability may improve the variability-based AGN selection.

A weakness of our difference image LCs is relatively large uncertainties on flux measurements that resulted from aperture photometry, while the recalibrated photometric data set (Ivezić et al. 2007) uses PSF magnitudes providing more precise photometric LCs at faint magnitudes. Several features, probably caused by lower $\mathrm{S} / \mathrm{N}$ of difference image $\mathrm{LCs}$, are detected. For example, there is a shift of the $\chi_{\nu}^{2}$ distribution to smaller values for difference image LCs, disappearance of the bifurcation in the $\chi_{\nu}^{2}$ distribution for the PhotoQSO sample that is seen in the photometric LC analysis, and systematically smaller best fit $\log \tau$ values for the common-epoch difference image LCs. Since there is a significant change in the shape of $\chi_{\nu}^{2}$ distribution (rather than just a shift of the entire distribution), loosening the $\chi_{\nu}^{2}$ criterion for difference image LCs is not helpful to construct a clear sample of intrinsically variable sources. Instead, this will cause high contamination in the sample by non-variable/faint sources with variability dominated by larger photometric noise. Figure 14 shows a $u-g$ vs. $g-r$ CCD for the Pho-

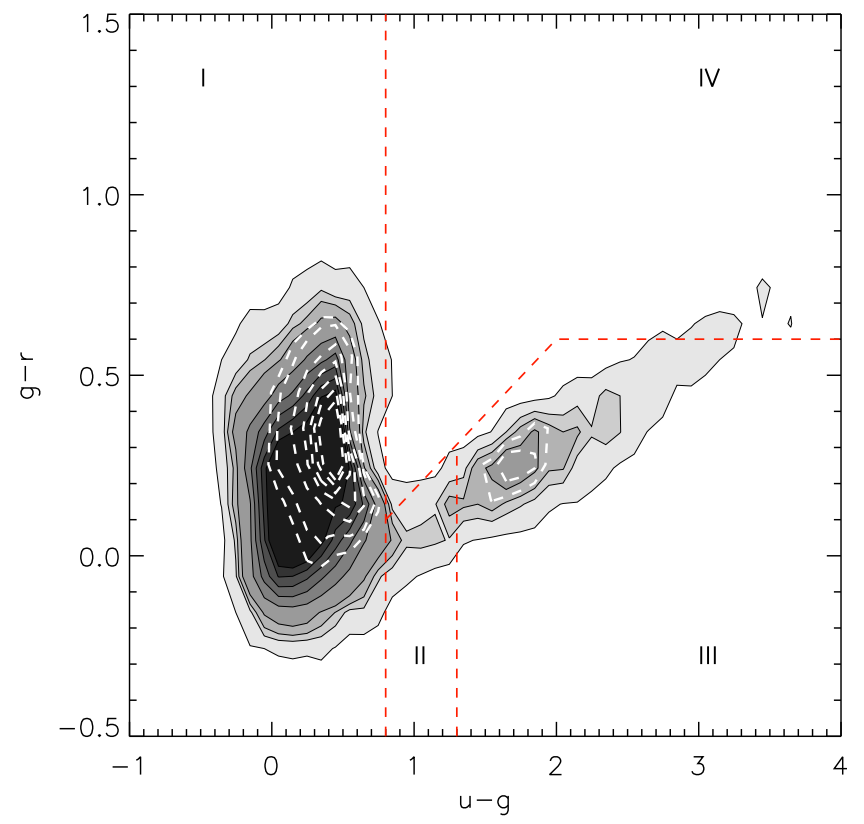

Fig. 14.- $u-g$ vs. $g-r$ CCD for the PhotoQSO sample. Black contours show the entire sample while white dashed contours represent sources with $\chi_{\nu}^{2}<3$.

toQSO sample. Sources with $\chi_{\nu}^{2}<3$ occupy the elongated part in Region I on the CCD. That no such feature is seen in the SpecQSO or the VarSrc samples implies that these sources are mostly non-variable. Visual inspection of their spectra in the SDSS DR9 (Ahn et al. 2012) indicates many of them are actual contaminants such as stars and galaxies or else are highly absorbed/reddened quasars.

However, we are able to minimize the effect of large uncertainties in difference image LCs on AGN selection by imposing appropriate selection criteria, which take systematic differences in the SF parameters into account (see Section 4.5). Furthermore, we stress that the SDSS is not optimized for image differencing and the primary goal of this study is to lay some groundwork for new time domain surveys that will really focus on obtaining higher-quality difference image data.

\subsection{Identifying QSOs by Variability Seen in Difference Images}

\subsubsection{Completeness and Efficiency}

To evaluate our AGN selection based on optical variability seen in difference images and compare to MacLeod et al. (2011), we compute the completeness (the fraction of the recovered SpecQSO) for given selection cuts using well-constrained LCs in the SpecQSO catalog. MacLeod et al. (2011) limit their sample to bright sources $(i<19)$ when estimating the completeness and the efficiency (i.e., purity) for their selection cuts because the SpecQSO catalog is complete for $\mathrm{i}<19$ (Table 2 in MacLeod et al. 2011). The completeness is defined as percentage of recovered quasars out of the entire confirmed quasar and the efficiency is defined as percentage of confirmed quasars out of selected objects (i.e., purity).

For bright sources, we achieve a completeness of $82.2 \%$, 
$96.4 \%$, and $98.6 \%$ for $\log \tau \geq 2,1.5$, and 1 , respectively. The completeness for $\log \tau \geq 2$ is lower compared to MacLeod et al. (2011), since we have systematically smaller $\log \tau$ values due to the differences in the methodologies of estimating the SF parameters (the direct fitting vs. the DRW model; the mean offset of $0.121 \pm 0.066 \mathrm{dex})$ and the difference in flux measurements (PSF vs. aperture photometry; the mean offset in $\log \tau$ of $0.271 \pm 0.339$ dex). When considering these combined offsets, lowering the selection cut on $\log \tau$ to $\sim 1.5$ is reasonable and it allows a comparable completeness to that for $\log \tau \geq 2$ in MacLeod et al. (2011), which was 94\% (93\% after omitting the most outlying data point in each LC). In fact, our completeness $(96.4 \%)$ for $\log \tau \geq 1.5$ is more similar to that $(96 \%)$ of MacLeod et al. (2011) when they apply both $\log \tau \geq 1.5$ and $\Delta L_{\text {noise }}>10$ (where $\Delta L_{\text {noise }}$ is the log likelihood of a DRW solution minus the log likelihood of a white noise solution). This indicates that our LC robustness cuts, especially the $\operatorname{rms}$ of $\log \tau$ cut, functions as the constraint $\Delta L_{n o i s e}>10$ in that both of them gauge the suitability of the DRW model for a given LC and boost the completeness and efficiency of the AGN selection.

Figure 15 shows the distribution of the SF parameters for the entire SpecQSO (black solid) and for the control sample (red dashed), which represents either nonvariable sources or empty spots on the sky (i.e., white noise). Including faint quasars $(i \geq 19)$ drops the completeness to $56.7 \%, 81.2 \%$, and $91 . \overline{4} \%$ for $\log \tau \geq 2,1.5$, and 1 , respectively. Unlike bright sources, faint sources in the SpecQSO can have $\log \tau<1.5$. This is mainly because relatively large uncertainties due to aperture photometry make difference image LCs of fainter sources indistinguishable from those of non-variable sources. It leads to a shift of the distribution of fainter sources towards the non-variable region in the SF parameter space. To maintain a reasonably high completeness of $91.4 \%$, one can adopt $\log \tau \geq 1$ as an AGN selection criterion. This cut statistically separates quasars well from the control sample; over $99 \%$ of the control sample shows $\log \tau<$ 1. The completeness for various $\tau$ criteria are listed in Table 3 .

The VarSrc catalog contains 6,573 spectroscopically confirmed quasars ( $~ 70 \%$ of the SpecQSO sample). The large number of non-quasar contaminants in the VarSrc catalog allows us to assess the efficiency of the AGN selection based on their variability. The estimated selection efficiency here, however, is the lower limit due to incompleteness of the subsample of confirmed quasars in the VarSrc catalog even at the bright end. In terms of brightness, this subsample is between the bright and the entire SpecQSO samples given the cut at $g<20.5$. Thus, the completeness of this subsample for the same selection cut is also expected to be between those for the bright and the entire SpecQSO samples. The completeness and efficiency as a function of the minimum log $\tau$ and maximum $\log \hat{\sigma}$ are shown in Figure [16] and Figure 17. respectively. Completeness of $65 \%$ and efficiency of $78 \%$ can be achieved for $\log \tau \geq 2$ and $\log \hat{\sigma} \leq 4.15$. If we decrease the minimum $\log \tau$ cut to 1.5 while maintaining the cut on $\log \hat{\sigma}$, the completeness significantly rises to $86 \%$ while the efficiency (75\%) decreases only by a few percent. By lowering the minimum $\tau$ criterion, one can select many more quasars without adding many

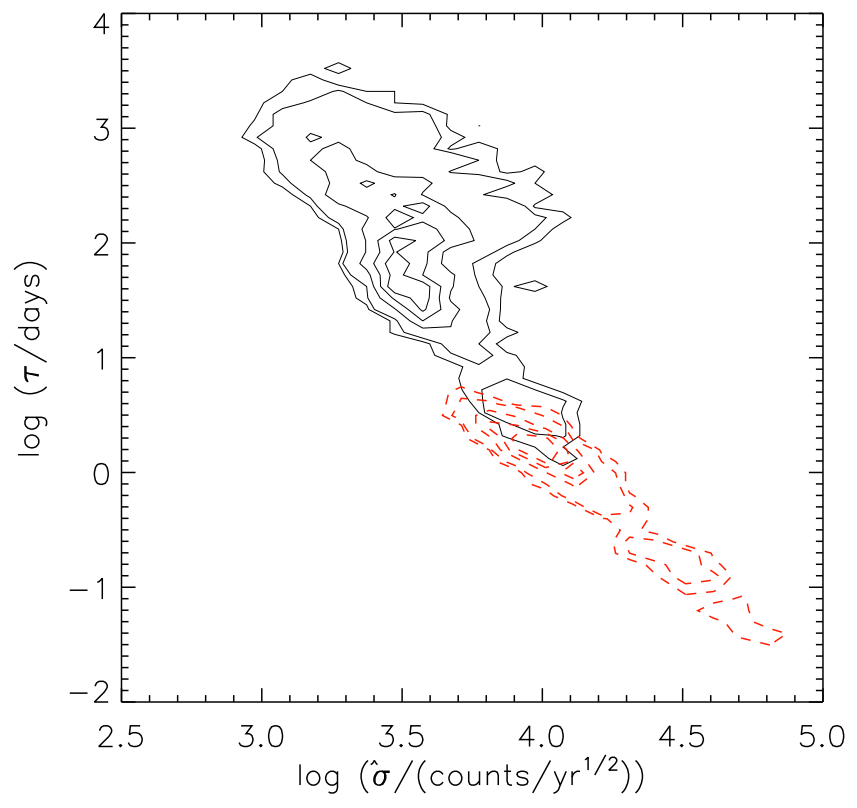

FIG. 15. - Characteristic damping timescale $\tau$ as a function of the driving amplitude of short-term variation $\hat{\sigma}$ for the SpecQSO sample (black solid) and for the control sample (red dashed). We draw contours at 5, 10, 30, 50, and 70 data points.

contaminants. When reducing the minimum $\log \tau$ cut further down to 1 , the completeness can increase to $93 \%$ with the efficiency of $71 \%$ and this selection criteria gives the maximum $\sqrt{C^{2}+E^{2}}$. The results are summarized in Table 4 .

We explore contaminants in detail to see if there are possible quasars that were not spectroscopically confirmed yet in the SDSS DR7 data set. We first select sources with $\log \tau \geq 1.5$, which is pretty conservative cut, that are not found in the SpecQSO catalog. Among 940 selected sources, 733 have colors consistent with lowredshift quasars (Region I on the $u-g$ vs. $g-r$ CCD ). We could retrieve 208 spectra from the DR9 data set and 4 spectra from spectroscopic follow-up with Apache Point Observatory DIS (MacLeod 2012), and find that 56\% of them are confirmed as quasars. If we assume that the fraction of quasars $(56 \%)$ remains the same, then the efficiency for the $\log \tau \geq 1.5$ cut is expected to increase by at least $10 \%$ for a spectroscopically complete sample.

When adopting $\log \tau \geq 1(1.5,2)$ as an AGN selection criterion, $66 \%(52 \%, 30 \%)$ of the PhotoQSO sample are classified as AGNs. A large fraction of sources $(\sim 40 \%)$ with $\log \tau<1$ show photometric $\chi_{\nu}^{2}<3$, indicative of observed variability dominated by photometric noise. These sources account for about $13 \%$ of the entire PhotoQSO sample. Thus, we can conclude that there are at least $\sim 13 \%$ of possible contaminants in the PhotoQSO based on our variability analysis. There are a few sources (21 objects) whose characteristic damping timescales are in the range of 1 to 10 days and that are bright in the X-ray. They actually show significant evidence for intrinsic optical variability (i.e., $\chi_{\nu}^{2} \geq 3$ and $\Sigma \geq 0.05$ ), but are not found in the SpecQSO catalog. Some of them could be faint typical AGNs with relatively 


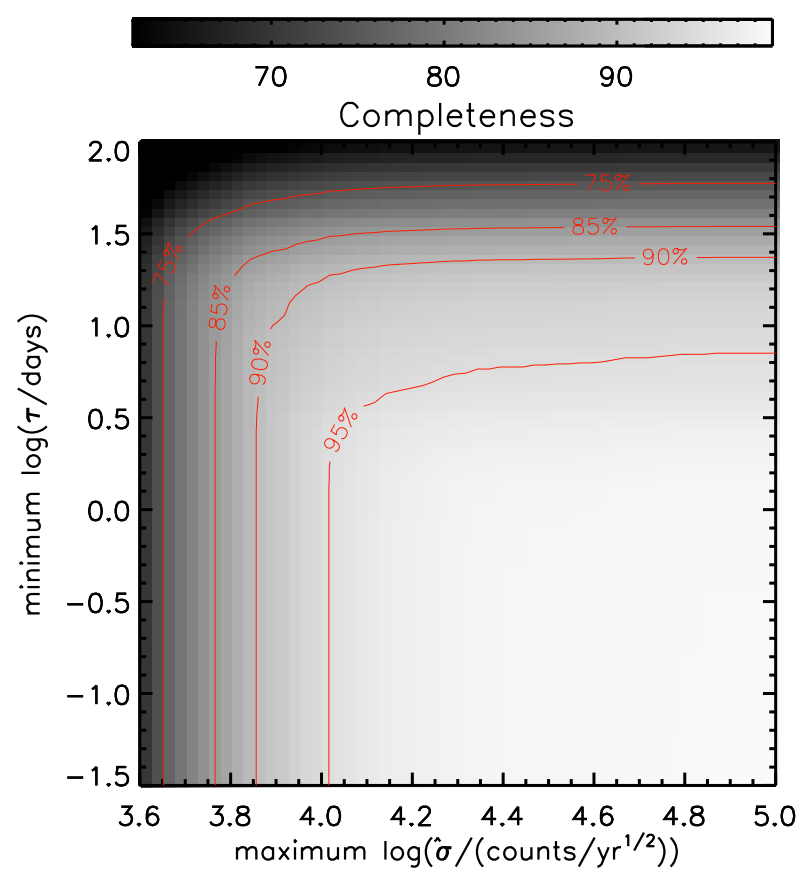

FIG. 16. - The completeness for AGN selection criteria for the VarSrc sample.



FIG. 17.- The efficiency for AGN selection criteria for the VarSrc sample.

short damping timescale. Another possible explanation for these objects is that they might be blazars.

\subsubsection{Blazar Candidates}

Our discussion of AGN variability thus far has focused primarily on the optical variability characteristics of the thermal disk continuum emission from typical Type I quasars. However, some rare AGNs (blazars) are instead dominated in their optical emission by a relativistic jet aligned with the line of sight (Blandford \& Rees 1978); these jet-dominated objects are known to be even more variable than typical quasars due to the effects of relativistic beaming. Ruan et al. (2012) have shown that blazar optical light curves are also well-described as a DRW process, but with smaller $\tau$ and larger $\hat{\sigma}$ than typical quasars. These blazars should thus stand out as extreme variability outliers in our difference image LCs, allowing for variability-based identification. As a test of our ability to detect this non-thermal jet emission through optical variability in our difference image LCs, we select extreme variability objects with $\log \hat{\sigma}>4.2$ and $1<\log \tau<3$ in the VarSrc sample. These cuts will select objects with smaller $\tau$ and larger $\hat{\sigma}$ than the vast majority of confirmed quasars (e.g., in the SpecQSO sample in Figure 15), and are consistent with emission affected by relativistic beaming in an aligned jet (Ruan et al. 2012).

Massaro et al. (2011) showed that blazars detected in Wide-field Infrared Survey Explorer (WISE; Wright et al. 2010) all-sky survey tend to lie along a narrow 'WISE blazar strip' on WISE CCD. To gauge whether our highly-variable objects are of blazar origin, we positionally match the VarSrc sources to the WISE all-sky catalog, using a $3^{\prime \prime}$ matching radius. Figure 18 shows the WISE W1-W2 vs. W2-W3 filter CCD of these matches that have $\mathrm{S} / \mathrm{N}>3$ in the WISE W1 [3.4 $\mu \mathrm{m}]$, W2 [4.6 $\mu \mathrm{m}]$, and W3 [12 $\mu \mathrm{m}]$ bands. Highly variable sources in this sample $(\log \hat{\sigma}>4.2$ and 1 $<\log \tau<3$ ) are highlighted as red star symbols. We also show the WISE blazar strip by overplotting the results of a Gaussian Kernel Density Estimate using the 3,021 known blazars in the Roma-BZCAT blazar catalog (Massaro et al. 2009) which matched to WISE sources with $\mathrm{S} / \mathrm{N}>3$ in W1, W2, and W3. In Figure 18, the cloud of variable sources (black points approximately centered at $(\mathrm{W} 1-\mathrm{W} 2, \mathrm{~W} 2-\mathrm{W} 3)=(1.1$, $3.1)$ are mostly AGN, while sources with W1-W2 < 0.5 tend to be stars and galaxies. Many of the highly variable sources highlighted appear to be AGN, as well as many stars. Although the WISE blazar strip is relatively narrow, much of it lies directly on top of the cloud of typical (non-jet dominated) AGNs, making it difficult to determine whether a source near the WISE blazar strip is dominated by non-thermal jet emission. The relatively low efficiency of blazar selection using the WISE blazar strip (especially for the flat-spectrum radio quasar sub-class of blazars) has been noted previously (Ruan et al. 2012). Nevertheless, it is intriguing that the positions of the highly-variable sources with W1-W2 $\geq 0.5$ do not appear to sample the distribution of the more numerous quasars in the background, but rather seem to lie preferentially closer to the WISE blazar strip. Thus, it is possible that many of these sources are blazars, with blazar-like WISE colors and extreme optical variability. In fact, one third of them are found in the Stripe 82 radio catalog by Hodge et al. (2011). While it is clear that WISE colors alone do not efficiently separate blazars from non-jet dominated quasars due to their strong overlap in the WISE CCD, our inclusion of additional variability information can greatly help in narrowing down the candidates. 


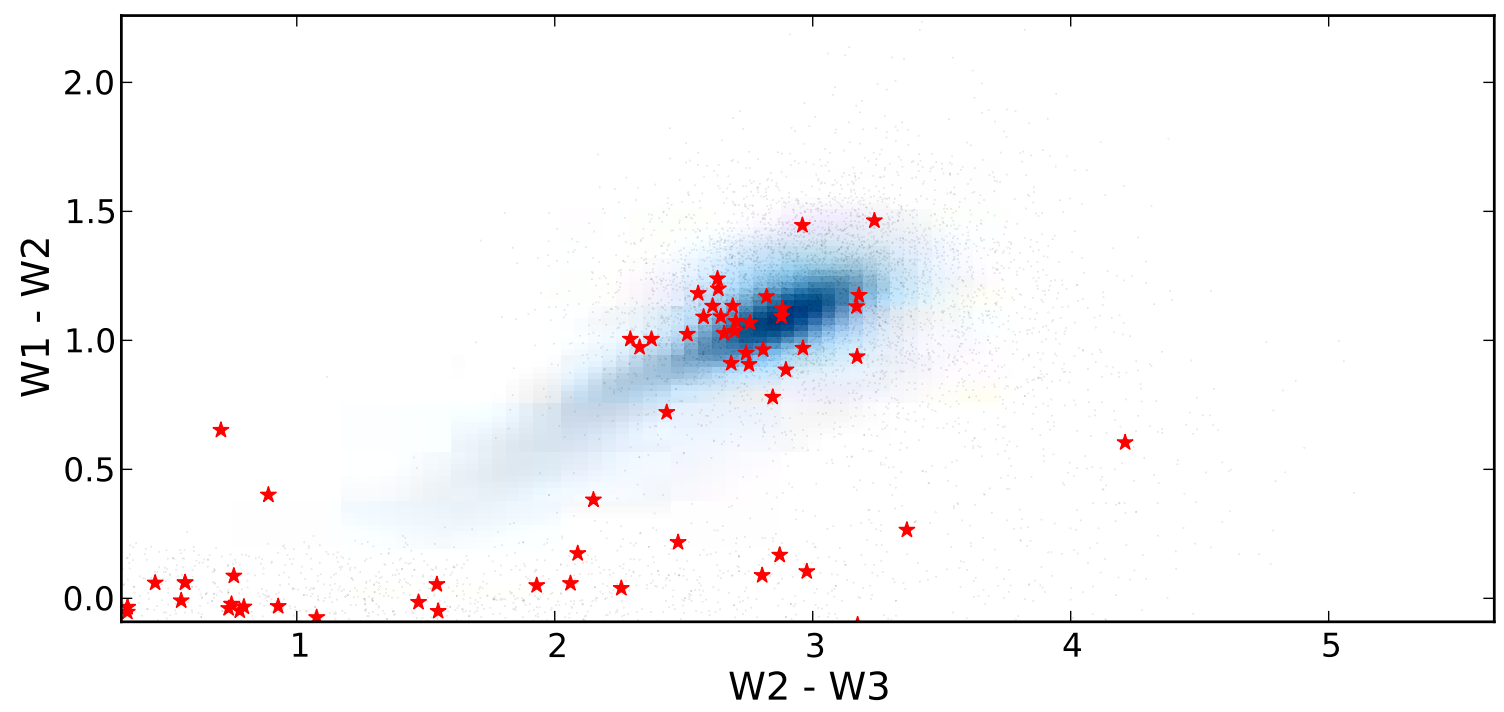

FIG. 18. - WISE W1-W2 vs. W2-W3 CCD of all VarSrc sources passing the robustness cuts, matched to WISE sources with $\mathrm{S} / \mathrm{N}>3$ in the WISE W1 $[3.4 \mu \mathrm{m}]$, W2 $[4.6 \mu \mathrm{m}]$, and W3 [12 $\mu \mathrm{m}]$ bands (black points). Highly variable sources with $\log \hat{\sigma}>4.2$ and $1<\log \tau<3$ are highlighted as red star symbols. A Gaussian Kernel Density Estimate of the location of the WISE blazar strip is shown in blue. Sources with W1 - W2 $<0.5$ tend to be stars and galaxies.

\subsection{Identifying AGNs in X-Rays}

Figure [19] shows the distribution of the SF parameters for X-ray selected optically-unresolved sources before applying the cut on $\operatorname{rms}$ of $\log \tau$. The data are color coded by their magnitude in $g$-band. Most sources that are fainter than $g \simeq 21.5$ tend to have short damping timescales $(\log \tau<1)$. Some of them are intrinsically faint in the optical, and some of them are faint due to obscuration (e.g., Type II AGN). A strong magnitude dependence of the completeness of variabilitybased AGN selection is well-known (e.g., Kozłowski et al. 2010). Given that photometric noise becomes large and uncorrelated between observations for faint sources, reported small $\tau$ values for faint AGNs may be spuriously induced. If AGNs are not bright enough in the optical, their optical LCs could be neither recognized as typical AGNs nor well constrained with DRW model (i.e., spurious small $\tau$ with $\operatorname{rms}$ of $\log \tau>0.15$ ). This implies that applying the same AGN selection cuts regardless of magnitude can result in missing many faint AGN candidates. According to visual inspection of spectra of objects with $\log \tau \geq-1,95 \%$ are indeed classified as QSOs (only a small fraction of contamination by active late-type stars). A much higher contamination rate is found in sources with $\log \tau<-1$. Therefore, we conclude that X-ray detection provides additional power to efficiently discriminate true faint AGNs from the contaminants even down to $\log \tau \geq-1$, the regime where it is usually impossible to separate true faint AGNs from objects dominated by photometric noise solely based on the variability properties. Note that we do not claim that these short $\tau$ values are related to physical timescales of any accretion processes. Even though photometric noise for faint sources will be improved by deep imaging surveys and accurate PSF photometry, X-ray detection as an additional con-

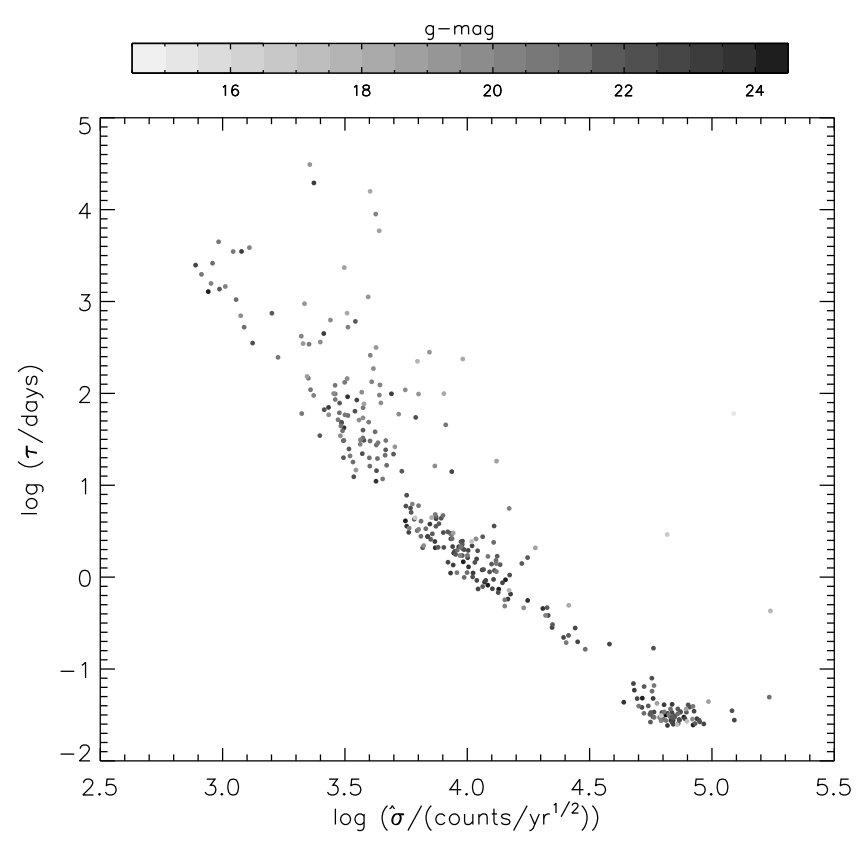

FIG. 19.- Distribution of the SF parameters for X-ray selected point-like sources, color coded by $g$ magnitude. Faint sources tend to have shorter characteristic timescales. Although large and uncorrelated photometric noise may spuriously induce apparent short timescale variability in optically faint objects, X-ray detection provides additional power to efficiently discriminate true faint AGNs from contaminants (see discussion in Section 4.6).

straint would still be very helpful to finding faint AGNs, and thus boosting the selection completeness and efficiency.

To probe the origin of AGN optical variabil- 
ity, correlation between optical/UV and X-ray variability has been studied in terms of both time lag and relative variability amplitude between two bands (e.g., Clavel et al. 1992; Peterson et al. 2000; Edelson et al. 2000; Nandra et al. 2000; Shemmer et al. 2003; Uttlev et al.|2003; Arévalo et al. 2009; Kelly et al. 2011; Cameron et al. 2012). However, there has been debate over the nature of optical variability since some AGNs (e.g., NGC 5548 or NGC 4395) show a strong correlation, with a high cross-correlation coefficient, between the two bands on both short and long-term timescales whereas some show no clear correlation at all (e.g., NGC3516 or NGC 7469). In some other AGNs, the two bands are uncorrelated on shorter timescales while they are correlated on longer timescales, and vice versa (e.g., NGC 4051 or Ark 564). This puzzling variety in observed relations between optical/UV and X-ray variability implies that the process is too complex to be explained solely by a single model even in a single AGN, such as reprocessing of X-rays (Guilbert \& Rees 1988), propagating of intrinsic random fluctuation in the accretion rate inward through accretion flow (Arévalo et al. 2008) and Compton up-scattering of optical/UV photons (Haardt \& Maraschi 1991). Lack of large samples that are simultaneously observed in both bands with high cadence over long timespans have made it hard to understand their connection. Alternatively, it may suggest that an AGN accretion disk fundamentally requires all combined effects of these mechanisms at the same time.

We search for correlations between the optical variability and X-ray properties, such as spectral shape and luminosity. The hardness ratio, $\left(\mathrm{F}_{H_{a r d}}\right.$ $\left.\mathrm{F}_{\text {Soft }}\right) /\left(\mathrm{F}_{\text {Soft }}+\mathrm{F}_{\text {Medium }}+\mathrm{F}_{\text {Hard }}\right)$, is used as an approximation of the X-ray spectral shape (Evans et al. 2010). Here, $\mathrm{F}$ denotes the aperture photon flux in each $\mathrm{X}$ ray band (soft:0.5-1.2 keV, medium:1.2-2.0 keV, and hard:2.0-7.0 keV). For objects having redshift information in DR9, we compare the optical variability to the $\mathrm{X}$-ray properties in the rest frame in Figure 20. We find no correlations between hardness ratio and either $\mathrm{SF}_{\infty}$ or $\tau$ (top panels). No noticeable correlations between the optical variability and hardness ratio indicate that the X-ray spectral shape might be associated only with the rapid variability in X-ray (König et al. 1997), which would not be well reflected in optical LCs if we consider the reprocessing mechanism. The correlations, if they indeed exist, also could be washed out because the optical variability properties are drawn from multiepoch observations while the hardness ratio is measured from a single-epoch observation. Grupe et al. (2010) studied 92 bright soft X-ray selected AGNs and they do not detect significant level of X-ray spectral variability on timescales of a year or two, indicative of no correlation with long-term optical variability. However, Nandra et al. (2000) find that the X-ray spectral shape is positively correlated with the UV flux in NGC 7469, supporting Comptonization, although UV and X-ray fluxes are uncorrelated. We do not see any statistically meaningful correlations between the optical variability and Xray luminosity, thus no trend that X-ray luminous AGNs are more variable (MacLeod et al. 2010; Butler \& Bloom 2011; Young et al. 2012).

\section{VARIABILITY OF RESOLVED SOURCES}
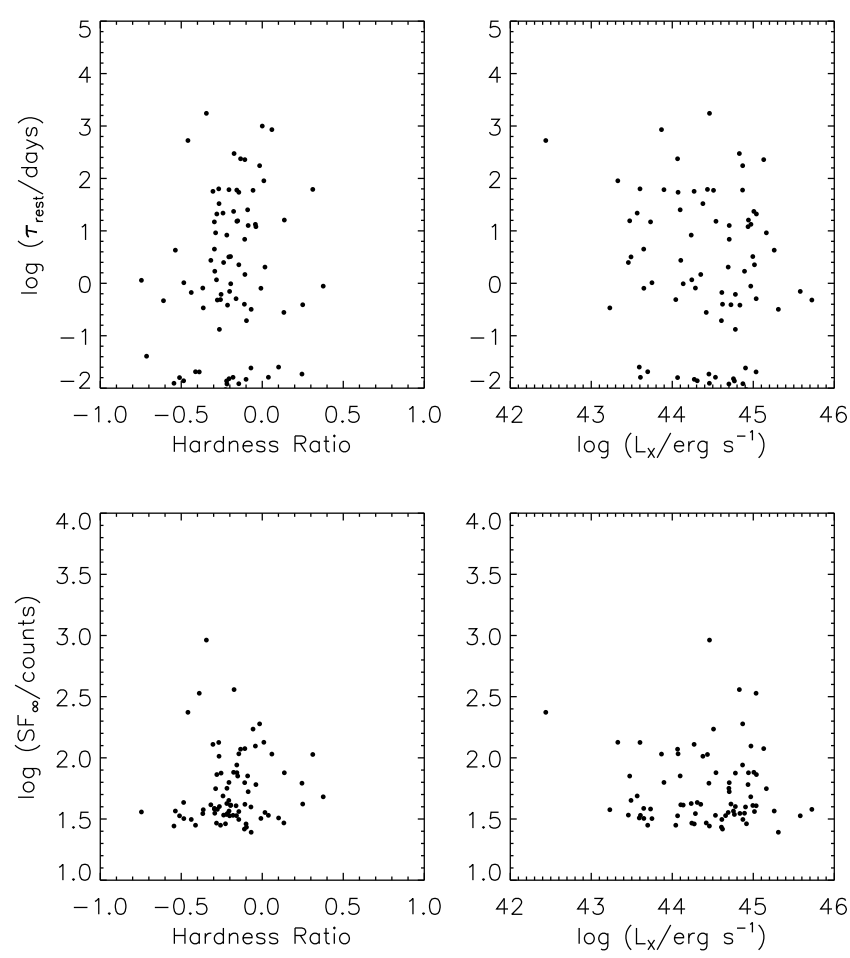

FIG. 20.- Comparison between the rest-frame SF parameters and $\mathrm{X}$-ray properties.

\subsection{The Optical Variability and Spectral Properties}

To describe the whole population of AGNs and its evolution over cosmic time, compiling a sample of extended (i.e., host-dominated) AGNs, which are usually omitted from photometric or even from spectroscopic catalogs of QSOs/AGNs, is necessary. While current optical variability surveys focus on unresolved bright sources, image differencing will be the fundamental means of distinguishing variability in extended sources for upcoming surveys. Since image differencing only leaves signal from variable regions, we are able to identify AGN activity blended with extended host galaxies, where traditional photometric selection is challenged. Close pairs of AGN also can be identified without fiber collisions that complicate multi-object spectroscopic searches.

Previous optical variability studies of extended sources have been accomplished using high angular resolution images to minimize the effect of the host galaxy light on the nuclear variability (e.g., Bershady et al. 1998; Klesman \& Sarajedini 2007; Trevese et al. 2008; Boutsia et al. 2009; Cameron et al. 2012). However, it was impossible to perfectly remove light contamination by surroundings from flux measurement of the nuclear activity. Furthermore, most previous studies utilized imaging data monitored for less than 2 years with small coverage of the sky.

Our final goal in this paper is to explore optical properties of X-ray detected optically-extended sources, and to evaluate variability selection as a tool for distinguishing AGNs from star-forming galaxies and other types of extended sources that can contaminate AGN catalogs. 
To do this, we first construct a subsample of 537 candidates of extended AGN from the XRaySrc catalog by removing objects that are unresolved in the optical when the optical information is available. Since most of X-ray detected AGN candidates are faint in the optical, only 222 objects ( $41 \%$ of the subsample) have optical counterparts. More than half of them are fainter than $g=21.5$, and their magnitude distribution is similar to that of Xray detected unresolved sources. Based on their optical variability, $\sim 67 \%$ of them can be selected as AGN candidates $(\log \tau \geq-1$; see Section 4.6). With a more conservative cut, $\log \tau \geq 0$, half of them can be selected as AGN candidates. None of them are suspected as lensed QSO candidates (Lacki et al. 2009).

We investigate available optical spectra of 89 (out of 222) optically-extended AGN candidates by simple visual inspection. The majority of them show clear emission lines. According to their spectral properties, such as line ratio and width, the sample of 89 AGN candidates consists of various types of AGNs (66\%) as well as star-forming galaxies (11\%), other emission-line galaxies $(6 \%)$, X-ray bright optically inactive galaxy (XBONG) candidates $(6 \%)$, composite galaxies (4\%), normal galaxies (4\%), and unknown objects (3\%). Among AGNs, we further divide their spectral class in 5 sub-classes: QSO, Type I/II, narrow-line Seyfert1 (NLS1), and Seyfert 1.9. Classification is performed based on the traditional definitions of each sub-class. In Type II spectra, only the narrow lines are present. NLS1 galaxies are known to have smaller black hole masses that are accreting very close to the Eddington rate, thus bright in the X-ray, and exhibit lower amplitudes of optical variability (Ai et al. 2013). In NLS1, a strong FeII line is expected. NLS1 is also characterized by weak [OIII] line $([\mathrm{OIII}] / \mathrm{H} \beta<$ $3)$. Seyfert 1.9 galaxies have an emission line properties such that $\mathrm{H} \alpha$ line is the only broad line component in their spectra (Osterbrock 1981). The combined QSO and Type I sample, both featured in broad line components, makes up $55 \%$ of our AGN sample. The fractions of Seyfert 1.9, Type II, and NLS1 in the sample are 26\%, $10 \%$, and $9 \%$, respectively. In Figure 21, we provide some examples of representative optical images and spectra for each type.

The characteristic damping timescales of most AGNs (91\%) are longer than $\log \tau=-1$ while half of starforming galaxies have $\log \tau<-1$. Therefore, optical variability will be a very efficient tool for separating AGNs from star-forming galaxies without emission line ratios diagnostics. We find some emission line galaxies showing spectral properties that are inconsistent with those of both star-forming galaxies and AGNs. Although the origin of their emission lines is ambiguous, it is unlikely that those emissions come from AGN activity based on their extremely short damping timescales $(\log \tau<-1)$. Likewise, in composite galaxies where star formation coexists with AGN activity suggesting a connection between star formation and AGN (e.g., Heckmas et al. 1997; Kewley et al. 2006; Davies et al. 2007), optical variability enables us to pinpoint AGN activity from composite galaxies that are ambiguous in the Baldwin-Phillips-Terlevich (BPT) diagnostics (Baldwin et al. 1981; Kewley et al. 2006). With the aid of optical variability information, we suggest that all our composite galaxies are AGN activity dominated.
In the sample of 89 AGN candidates, there are contaminants by apparently normal (i.e., no emission lines) galaxies. Galaxies that are luminous in the X-ray but exhibit normal optical properties could be either heavily obscured AGNs (e.g., XBONGs candidates), accreting in a radiatively inefficient way, or true normal galaxies in X-ray emitting clusters or groups. In any cases, Xray detection, as an additional constraint, is required to compile a complete sample of AGN candidates.

We find 6 Type II AGN candidates. They are characterized by narrow emission lines without broad components, high-ionization line ratios, and hard X-ray spectra. No overlaps are found between our Type II candidates and a list of Type II AGN candidates from Zakamska et al. (2003). According to the unification model, none or very low level of variability is expected for Type II AGNs due to a highly obscured accretion disk from viewing angles (e.g., Yip et al. 2009). However, we detect non-negligible amount of optical variability in 2 of our Type II AGN candidates on long-term timescales consistent with typical or faint AGN. They could be Type II AGNs without severe attenuation either due to less dusty torus or marginal viewing angles. Alternatively, they could be 'naked' Type I AGNs that lack broad line regions for some reason without signs of significant intrinsic absorption (Hawkins 2004).

For the first time, we conduct a systematic optical variability analysis for extended sources using a large difference image data set. Difference images allow us to reveal several different types of AGNs blended in extended sources, including Type II. However, it is impossible to take full advantage of such a large data set since there are observational (low $\mathrm{S} / \mathrm{N}$ ) and photometric (aperture photometry) limitations in this study.

\section{SUMMARY AND CONCLUSIONS}

We quantify LCs of AGN candidates in Stripe 82 and identify AGNs based on their optical $g$-band variability. Our AGN candidates are sub samples of spectroscopically confirmed quasars (SpecQSO), photometrically selected quasars using optical colors (PhotoQSO), variable point sources (VarSrc), and X-ray detected sources (XRaySrc). To construct a photometric LC for an individual source, we use the recalibrated photometric data set of Ivezić et al. (2007). We also construct difference image LCs by conducting a new image differencing and performing forced aperture photometry on all difference images. Although our difference image LCs have lower $\mathrm{S} / \mathrm{N}$ compared to their counterpart photometric LCs due to aperture photometry, they have, on average, 20 more reliable data points by virtue of forced photometry on all difference images. For each LC, we estimate the standard deviation $(\Sigma)$ and reduced $\chi^{2}\left(\chi_{\nu}^{2}\right)$ and confirm that most AGNs show intrinsic optical variability. We also characterize each LC with a damping timescale $(\tau)$ and a variability amplitude ( $\hat{\sigma}$ for short-term or $\mathrm{SF}_{\infty}$ for longterm). These SF parameters are derived in two different ways:(1) by directly fitting the first-order SF for the DRW (Equation 5) to all empirical $\mathrm{SF}(\Delta t)$ data points, and (2) by modeling individual LCs as a DRW. We show that there is a linear relationship between the best-fits from the both methods, with a systematic difference in $\tau$ values in that those estimated from the simple direct fitting are smaller. We explore the impact of $\mathrm{S} / \mathrm{N}$ and 
(a) QSO

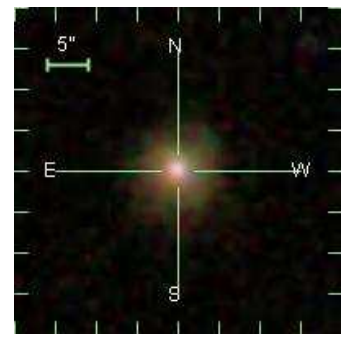

(c) Type II

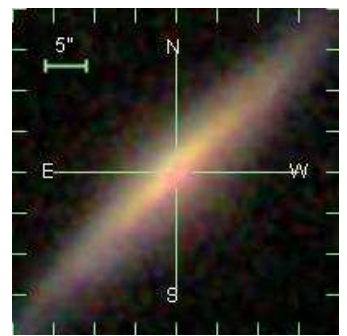

(e) Seyfert1.9

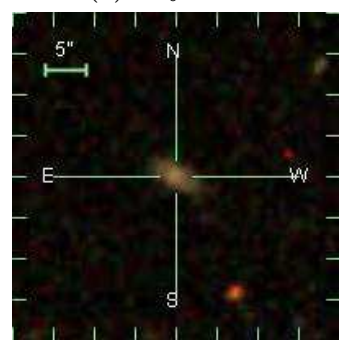

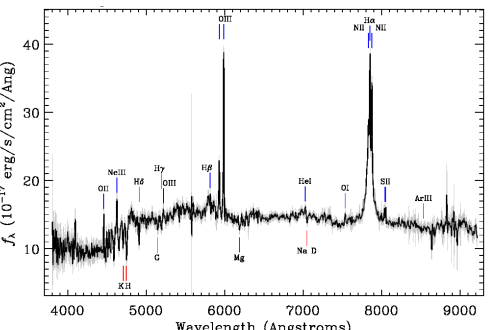
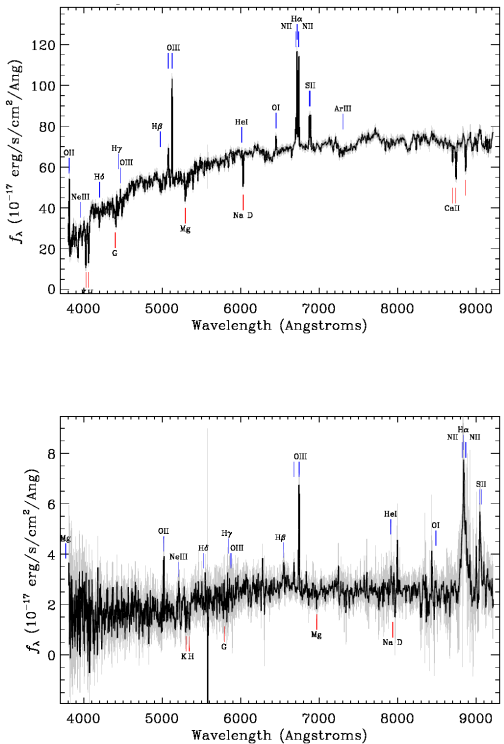

(b) Type I
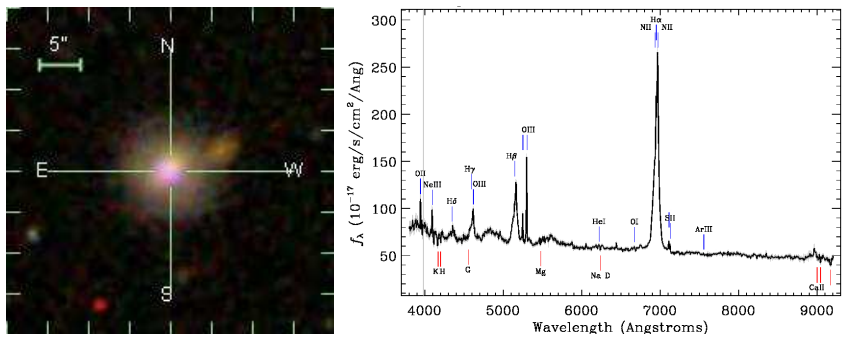

(d) NLS1
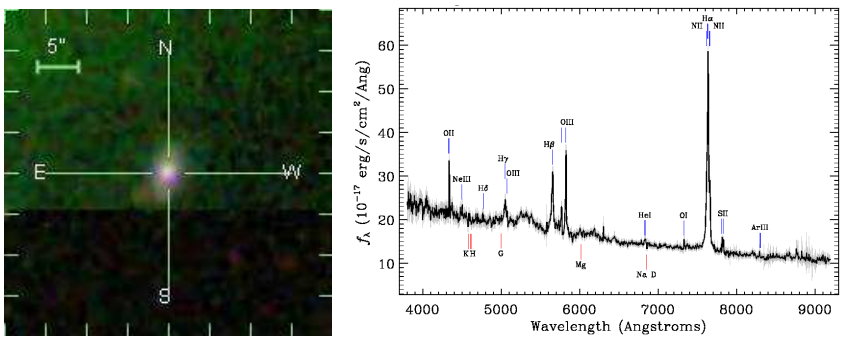

(f) SF galaxy
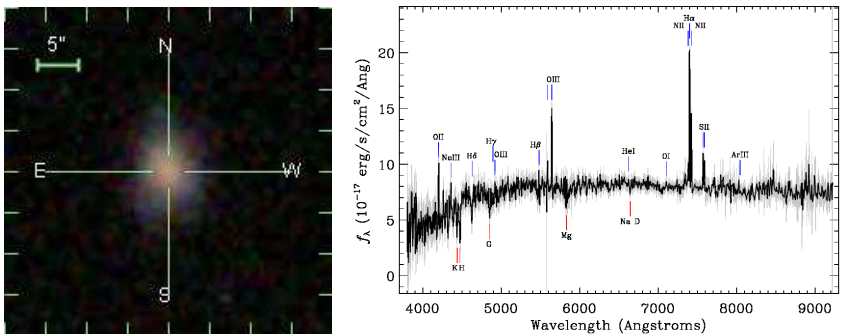

FIG. 21.- Examples of images and spectra for some X-ray detected optically-extended sources.

the number of data points in a $\mathrm{LC}$ on the $\mathrm{SF}$ parameter estimation. To provide a principled method for dealing with outliers in a LC and gauge how well the LC is described as the DRW, we perform jackknife resampling for each LC. For X-ray detected sources, we explore the relationship between the optical variability and the $\mathrm{X}$ ray properties and also quantify the optical variability of extended sources in large survey for the first time. Principal findings in our study are as follows:

1. We are able to select AGNs based on the optical variability detected in difference images with the high completeness and efficiency. For bright sources $(i<19)$, we achieve $\mathrm{C}=98.6 \%, 96.4 \%$, and $82.2 \%$ for $\log \tau \geq 1.0,1.5$, and 2.0 criteria, respectively. With the sample including fainter sources $(g<20.5)$ as well, we achieve $\mathrm{C}=93.4 \%$ and $\mathrm{E}=$ $71.3 \%$ for selection cuts on $\log \tau \geq 1.0$ and $\log \hat{\sigma} \leq$ 4.15 by maximizing $\sqrt{C^{2}+E^{2}}$.

2. We find some blazar candidates that are highly variable, with shorter $\tau$ than typical AGNs. One third of highly variable sources with IR colors consistent with known blazars are also radio detected.
3. Since optical counterparts of the majority of Xray detected sources are faint $(g>21)$, their difference image LCs are mostly dominated by photometric noise that may spuriously induce apparent short damping timescales, mimicking those of non-variable objects. According to spectral classification, however, contamination rate by non-AGNs rapidly drops at $\tau>0.1$ days (less than $5 \%$ ). Thus, we are able to efficiently select faint AGNs from contaminants with a combination of additional Xray information and a loose cut on $\tau$.

4. No significant relationships are found between the rest-frame SF parameters and X-ray properties, such as hardness ratio and X-ray luminosity.

5. Among X-ray detected optically-extended sources, we identify various types of AGNs including Type I, Type II, Seyfert 1.9, and NLS1 based on visual inspection of their optical spectra.

6. For composite galaxies, we expect that the optical variability characteristics provides a powerful tool 
to determine whether a dominant emission source is AGN activity or star-forming.

7. Contrary to the expectation from the unified model, one-third of our Type II AGNs show nonnegligible variability on long-term timescales that are consistent with typical Type I AGNs.

This study is an initial investigation of the effectiveness of difference image-based AGN classification. Future work can extend our analysis in several ways: (1) generating difference images with PSF-matched templates, (2) conducting PSF photometry on difference images, and (3) exploring all extended sources in Stripe 82. This will eventually lead to significant improvements in our understanding of properties of faint AGNs.

The authors thank Christopher S. Kochanek for helpful discussion. Support for this work was provided by the National Aeronautics and Space Administration through Chandra Award Numbers AR9-0015X, AR0-11014X, and AR2-13007X, issued by the Chandra X-ray Observatory Center, which is operated by the Smithsonian Astrophysical Observatory for and on behalf of the National Aeronautics Space Administration under contract NAS803060.

Funding for the SDSS and SDSS-II has been provided by the Alfred P. Sloan Foundation, the Partic- ipating Institutions, the National Science Foundation, the U.S. Department of Energy, the National Aeronautics and Space Administration, the Japanese Monbukagakusho, the Max Planck Society, and the Higher Education Funding Council for England. The SDSS Web Site is http://www.sdss.org/.

The SDSS is managed by the Astrophysical Research Consortium for the Participating Institutions. The Participating Institutions are the American Museum of Natural History, Astrophysical Institute Potsdam, University of Basel, University of Cambridge, Case Western Reserve University, University of Chicago, Drexel University, Fermilab, the Institute for Advanced Study, the Japan Participation Group, Johns Hopkins University, the Joint Institute for Nuclear Astrophysics, the Kavli Institute for Particle Astrophysics and Cosmology, the Korean Scientist Group, the Chinese Academy of Sciences (LAMOST), Los Alamos National Laboratory, the Max-Planck-Institute for Astronomy (MPIA), the MaxPlanck-Institute for Astrophysics (MPA), New Mexico State University, Ohio State University, University of Pittsburgh, University of Portsmouth, Princeton University, the United States Naval Observatory, and the University of Washington.

This research made use of data obtained from the Chandra Source Catalog, provided by the Chandra Xray Center (CXC) as part of the Chandra Data Archive.

APPENDIX

CATALOGS

Spectroscopically Confirmed Quasar Catalog

Schneider et al. (2010) found 105,783 spectroscopically confirmed quasars from the SDSS DR7. They were selected from quasar candidates (mainly color-selected) based on their spectral properties and a luminosity criterion $\left(M_{i}<\right.$ $-22.0)$. The redshift distribution of the sample covers a wide range from 0.065 to 5.46 , and has a median of $z \approx 1.5$ (see Figure 5 in Schneider et al.|2010). A total of 9,519 spectroscopically confirmed quasars ("SpecQSO") are located in Stripe 82 and approximately $97.2 \%$ of them have matched photometric LCs from our SDSS photometry data set, recalibrated by Ivezić et al. (2007) for unresolved sources in Stripe 82 (Section 3.1). Even though this sample does not include very faint objects (having a mean $g$ magnitude of 19.9), it is very useful to study AGN variability because it is a bona fide quasar sample. Since the SpecQSO sample are bright and bona fide, many of these ( $\sim 70 \%)$ were also identified as optically variable sources by Sesar et al. (2007) (Section A.3). In the $u-g$ vs. $g$-r diagram (top left panel in Figure 2), most SpecQSO occupy the bluer color portion (Region I) and some with high-redshift are most likely to occupy Region III.

\section{Photometrically Selected Quasar Catalog}

In contrast to the SpecQSO catalog, the catalog of 1,000,000 SDSS DR6 quasar candidates, photometrically selected based on their colors (Richards et al. 2009), contains fainter sources as well. The mean $g$ magnitude of this sample is about 1 mag fainter than the SpecQSO sample. Thus it can be used to examine the capability of the difference imaging method at the faint end. Even at the bright end, it has more sources $(\sim 1.6$ times more at $g<18)$ than the SpecQSO sample since there are no fiber collision issues. The expected completeness of this photometric quasar catalog to type I quasars is about $70 \%$ (Richards et al. 2009). The overall efficiency of their selection algorithm, which is much simpler compared to spectroscopic selection, reaches up to $\sim 70 \%$, while the efficiency for candidates flagged as "good" (i.e. a parameter "good" $\geq 0$, ranging from -6 to 6$)$ exceeds $90 \%$, especially at redshifts $(\mathrm{z} \leqslant 2.2)$ where color contamination is minimal. Of the $\sim 1,000,000$ objects in the catalog, 36,775 "good" quasar candidates ( "PhotoQSO") reside in Stripe 82 and also have photometric LCs. This is about 4 times larger than the SpecQSO sample, allowing for more quasar candidates even in Region III (top right panel in Figure 22). It is worth noting that the contours for the PhotoQSO sample in Region I are a bit elongated toward redder colors, which is not seen for the SpecQSO sample. This might result from contamination by other sources, and we investigate possible causes of this elongation in Section 4 . 


\section{Variable Source Catalog}

We use the Stripe 82 variable source catalog (Ivezić et al. 2007; Sesar et al. 2007). ${ }^{4}$ This catalog lists 67,507 variable point source candidates. They are selected by the following conditions:

- the number of observations in both the $g$ and $r$ bands $\geq 10$

- the root-mean-square (rms) scatter in both the $g$ and $r$ bands is $\geq 0.05$ mag

- the LC $\chi_{\nu}^{2}$ in both the $g$ and $r$ bands $\geq 3$ around the mean

- the median $g$ band mag is $<20.5$.

This catalog is dominated by the stellar locus stars (Region IV in Figure 2) and low-redshift quasars (Region I). Based on colors, $\sim 15 \%$ are expected to be low-redshift quasars $(\mathrm{z}<2)$ with some contamination from white dwarfs and binaries. The remaining sources are primarily main-sequence stars $(\sim 82 \%)$ with some additional contribution from RR Lyrae (Region II; 1.75\%) and high-redshift quasars (Region III; 1.15\%). Based on the coverage of the SDSS Stripe 82 template images (from which we generate difference images), we select 31,992 sources from the original list of 67,507 variable sources. This sample is referred to as "VarSrc", and all have photometric LCs. Since this VarSrc sample includes other types of variables than quasars, we are able study differences in the LCs' properties or SF parameters between quasars and other variable sources, such as stellar locus stars and RR Lyrae (Section 4).

\section{X-ray Source Catalog}

The Chandra Source Catalog (CSC; Evans et al. 2010) provides X-ray properties for 94,700 distinct point and compact sources with observed spatial extent $\leq 30^{\prime \prime}$. Although this catalog only contains point-like X-ray sources, their optical counterparts may be extended sources such as host-dominated type I AGNs and type II AGNs. By removing optical point sources from the catalog, we compile a subset of AGN candidates that are bright and compact in the X-ray, but possibly extended in the optical. For the first time, we explore the optical variability of these X-ray luminous and optically resolved sources in Section 5. Evans et al. (2010) uniformly processed all the mission data from pointed observations and derived useful quantities, including aperture photometry fluxes in five energy bands, hardness ratio, and spectral model fits. To minimize the number of spurious sources in the catalog, they accepted only 3-sigma detections and set the minimum flux significance threshold to $\sim 10$ photon counts in the broad band. This should lead to less than 1 false detection per image. There are about 1,900 X-ray sources within Stripe 82 ("XRaySrc").

To match the CSC with our photometric data set, we adopt a matching radius of $1^{\prime \prime}$, corresponding to about $>$ 95\% match probability based on the statistical analysis of CSC-SDSS DR7 cross-match pairs (Rots \& Budavári 2011). This yields only $\sim 640$ matches, mostly because their optical counterparts are extended sources rather than unresolved sources: recall that our Stripe 82 photometric data set does not include resolved sources (though in Section 5 , we consider additional optically-resolved, X-ray compact sources in difference images). In addition, some of their optical counterparts are too faint to be detected by SDSS since X-ray selection generally favors objects with higher redshift. The mean $g$ magnitude for matched sources are 21.33 (see Figure 1). This sample is fainter than any other catalogs used in this study (down to $g \simeq 25$ ).

Although less than half of the XRaySrc have photometric LCs, we are still able to characterize the optical variability of all XRaySrc using difference image LCs. If we can address well how the optical variability of X-ray detected objects is related to their X-ray properties, it may be possible to identify AGNs in X-rays with high confidence even if they are not detected in optical (see Section 4.6).

\section{FITTING IN FLUX VERSUS MAGNITUDE}

To be able to compare difference image results with the previous analyses, which were conducted in magnitude, we test if the same characteristic timescales are obtained for a given LC analyzed both in magnitudes and in fluxes by a given LC fitting methodology. To perform this test, we convert photometric LCs of the SpecQSO from magnitude to flux, and then estimate the SF parameters based on flux (as well as magnitude) using the direct fitting method. In Figure 22, we compare $\log \tau$ values for LCs in magnitudes to those for LCs in fluxes. For the direct fitting method, the slope is consistent with unity $(0.993 \pm 0.014)$ and the y-intercept with zero (0.016 \pm 0.036$)$. This good correlation indicates that the method is self-consistent against the linear and log scales at the observed low variability amplitudes. We also perform the same test on the DRW LC modeling with difference image LCs of the SpecQSO. In previous studies of quasar photometric variability, the DRW model has been applied only to photometric LCs in magnitude (e.g., Kelly et al. 2009; Kozłowski et al. 2010; MacLeod et al. 2010; Butler \& Bloom 2011). In the conversion of difference image LCs from flux to magnitude, we add the template flux of a source to its difference image LC data points and take $-2.5 \log$ of the total flux. However, we note that this conversion becomes unreliable in some cases where the absolute values of negative residual fluxes are comparable to or even greater than the template flux. This could happen if sources become intrinsically very faint. We exclude any LCs, which have at least one negative total flux, from this comparison. The test results using difference image LCs (Figure 22) show that the DRW LC modeling is less self-consistent (a slope of $0.922 \pm 0.019$ and an intercept of $0.323 \pm 0.042)$ compared to the direct fitting method. It has large scatter in

\footnotetext{
4 Available at http://www.astro.washington.edu/users/ivezic/sdss/catalogs/S82variables.html
} 

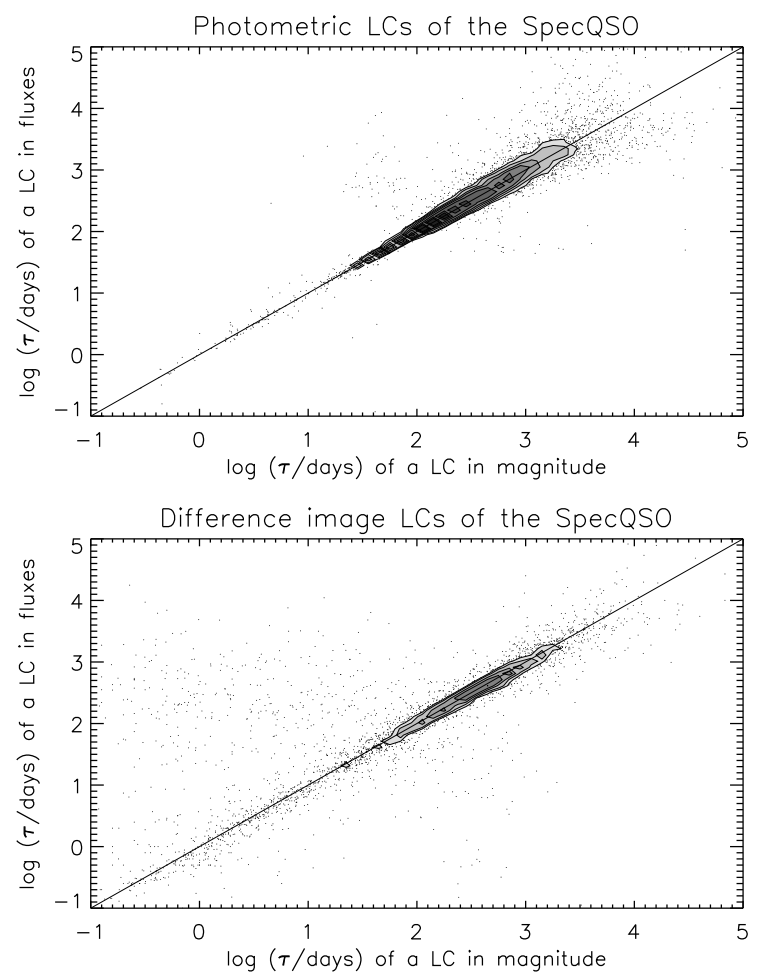

FIG. 22.- Top panel: A self-consistency test of the direct fitting method against the LC scales (linear vs. log) with photometric LCs. We overplot the one to one line (not a fit) to guide the eye. The result shows that the method is independent of the LC scales. Bottom panel: Same, but for the DRW LC modeling with difference image LCs.

the $\log \tau<1$ regime. This scatter is mostly responsible for the positive y-intercept. In fact, one can expect an even tighter correlation than the direct fitting case by excluding these scattered points. However, we find no good reasons to exclude them, as they are sources with well-constrained LCs (see the following subsection). Thus we conclude that the direct fitting method is insensitive to the linear versus log scales for observed LCs of QSOs/AGNs, and shows a better self-consistency than the DRW LC modeling, especially in terms of the intercept.

\section{REFERENCES}

Ahn, C. P., et al. 2012, ApJS, 203, 21

Ai, Y. L., et al. 2013, AJ, 145, 90

Alard, C., \& Lupton, R. H. 1998, ApJ, 503, 325

Alard, C. 2000, A\&AS, 144, 363

Annis, J., et al. 2011, ApJ, submitted (arXiv:1111.6619v2)

Arévalo, P., Uttley, P., Lira, P., Breedt, E., McHardy, I. M., \& Churazov, E. 2009, MNRAS, 397, 2004

Arévalo, P., Uttley, P., Kaspi, S., Breedt, E., Lira, P., \& McHardy, I. M. 2008, MNRAS, 389, 1479

Baldwin, J. A. 1977, ApJ, 214, 679

Baldwin, J. A., Phillips, M. M., \& Terlevich, R. 1981, PASP, 93, 5

Bauer, A., et al. 2009, ApJ, 696, 1241

Begelman, M. C., \& Celotii, A. 2004, MNRAS, 352, L45

Bershady, M. A., Trevese, D., \& Kron, R. G. 1998, ApJ, 496, 103

Blandford, R. D., \& Rees, M. J. 1978, in Proc. Pittsburgh Conference on BL Lac Objects, (Pittsburgh, PA: Univ. Pittsburgh), 328

Boutsia, K., Leibundgut, B., Trevese, D., \& Vagnetti, F. 2009, A\&A, 497, 81

Brandt, W. N., \& Hasinger, G. 2005 ARA\&A, 43, 827

Butler, N. R., \& Bloom, J. S. 2011, AJ, 141, 93

Cameron, D. T., et al. 2012, MNRAS, 422, 902

Cardamone, C. N. 2008, ApJ, 680, 130

Choi, E., Ostriker, J. P., Naab, T., Johansson, P. H. 2012, ApJ, 754,125

Clavel, J., et al. 1992, ApJ, 393, 113

Davies, R. I., Müller Sánchez, F., Genzel, R., Tacconi, L. J., Hicks, E. K. S., Friedrich, S., \& Sternberg, A. 2007, ApJ, 671, 1388

de Vries, W. H., et al. 2005, AJ, 129, 615
Di Matteo, T., Springel, V., \& Hernquist, L. 2005, Nature, 433, 604

Edelson, R., et al. 2000, ApJ, 534, 180

Evans, I. N., et al. 2010, ApJS, 189, 37

Frieman, J. A., et al. 2008, AJ, 135, 338

Fukugita, M., Ichikawa, T., Gunn, J. E., Doi, M., Shimasaku, K., \& Schneider, D. P. 1996, AJ, 116, 3040

Gibson, R. R., Brandt, W. N., \& Schneider, D. P. 2008, ApJ, 685, 773

Gibson, R. R., \& Brandt, W. N. 2012, ApJ, 746, 54

Grupe, D., Komossa, S., Leighly, K. M., \& Page, K. L. 2010, ApJS, 187, 64

Guilbert, P. W., \& Rees, M. J. 1988, MNRAS, 233, 475

Haardt, F., \& Maraschi, L. 1991, ApJ, 380, L51

Hawkins, M. R. S. 2004, A\&A, 424, 519

Heckman, T. M., et al. 1997, ApJ, 482, 114

Hodge, J. A., Becker, R. H., Richards, G. T., White, R. L. \& Zeimann, G. R. 2011, AJ, 142, 3

Hopkins, P., et al. 2007, ApJ, 669, 45

Hughes, P. A., Aller, H. D., \& Aller, M. F. 1992, ApJ, 396, 469

Ivezić, Z., et al. 2007, AJ, 134, 973

Ivezić, Ž., Tyson, J. A., Allsman, R., Andrew, J., Angel, R., \& the LSST collaboration. 2008, arXiv:0805.2366

Kaiser, N., et al. 2002, Proc. SPIE, 4836, 154

Kawaguchi, T., Mineshige, S., Umemura, M., \& Turner E. L. 1998, ApJ, 504, 671

Kelly, B. C., Bechtold, J., \& Siemiginowska, A. 2009, ApJ, 698, 895

Kelly, B. C., Sobolewska, M., \& Sobolewska A. 2011, ApJ, 730, 52 
Kewley, L. J., Groves, B., Kauffmann, G., \& Heckman, T. 2006, MNRAS, 372, 961

Kim, D.-W., Protopapas, P., Trichas, M., Rowan-Robinson, M., Khardon, R., Alcock, C., \& Byun, Y.-I. 2012, ApJ, 747, 107

Klesman, A., \& Sarajedini, V. 2007, ApJ, 665, 225

König, M., Staubert, R., \& Wilms, J. 1997, A\&A, 326, L25

Kozłowski, S., et al. 2010, ApJ, 708, 927

Lacki, B. C., Kochanek C. S., Stanek, K. Z., Inada, N., \& Oguri, M. 2009, ApJ, 698, 428

Law, N. M., et al. 2009, PASP, 121, 1395

Loeb, A., \& Barkana, R. 2001, ARA\&A, 39, 19

MacLeod, C. L., et al. 2010, ApJ, 721, 1014

MacLeod, C. L., et al. 2011, ApJ, 728, 26

MacLeod, C. L., et al. 2012, ApJ, 753, 106

MacLeod, C. L. 2012, PhD thesis, Univ. Washington

Massaro, F., et al. 2009, A\&A, 495, 691

Massaro, F., D'Abrusco, R., Ajello, M., Grindlay, J. E., \& Smith, H. A. 2011, ApJ, 740, L48

McHardy, I. 2010, in The Jet Paradigm, ed. T. Belloni (Lecture Notes in Physics, Vol. 794; Berlin: Springer), 203

Nandra, K., et al. 2000, ApJ, 544, 734

Peterson, B. M., et al. 2000, ApJ, 542, 161

Osterbrock, D. E. 1981, ApJ, 249, 462

Press, W. H., Rybicki, G. B., \& Hewitt, J. N. 1992, ApJ, 385, 404

Richards, G. T., et al. 2009, ApJS, 180, 67

Rots, A. H., \& Budavári, T. 2011, ApJS, 192, 8
Ruan, J. J., et al. 2012, ApJ, 760, 51

Sako, M., et al. 2008, AJ, 135, 348

Schmidt, K. B., Marshall, P. J., Rix, H.-W., Jester, S., Hennawi, J. F., \& Dobler, G. 2010 ApJ, 714, 1194

Schneider, D. P., et al. 2010, AJ, 139, 2360

Sesar, B., et al. 2007, AJ, 134, 2236

Sesar, B., et al. 2010, ApJ, 708, 717

Simonetti, J. H., Cordes, J. M., \& Heeschen, D. S. 1985, ApJ, 296,46

Shemmer, O., Uttley, P., Netzer, H., \& McHardy, I. M. 2003, MNRAS, 343, 1341

Springel, V., Di Matteo, T., \& Hernquist, L. 2005, MNRAS, 361, 776

Trevese, D., Kron, R. G., Majewski, S. R., Bershady, M. A., \& Koo, D. C. 1994, ApJ, 433, 494

Trevese, D., Boutsia, K., Vagnetti, F., Cappellaro, E., \& Puccetti, S. 2008, A\&A, 488, 73

Uttley, P., et al. 2003, ApJ, 584, 53

Vanden Berk, D. E., et al. 2004, ApJ, 601, 692

Wright, E. L., et al. 2010, AJ, 140, 1868

Yip, C. W., et al. 2009, AJ, 137, 5120

York, D. G., et al. 2000, AJ, 120, 1579

Young, M., et al. 2012, ApJ, 748, 124

Zakamska, N. L., et al. 2003, AJ, 126, 2125 
TABLE 1

List of CATAlogs for CANDidAte AGNs USEd in this StUdy

\begin{tabular}{c|c|c|c|c}
\hline \hline Object Type & Reference & $\begin{array}{c}\text { Num. of sources } \\
\text { in Stripe 82 }\end{array}$ & $\begin{array}{c}\text { Num. of sources } \\
\text { with photometric LC }\end{array}$ & $\begin{array}{c}\text { Num. of sources } \\
\text { with diffIm LC }\end{array}$ \\
\hline SpecQSO & Schneider et al. (2010) & 9,519 & 9,254 & 6,583 \\
PhotoQSO & Richards et al. (2009) & 36,884 & 36,775 & 24,596 \\
VarSrc & Ivezić et al. (2007) & 31,992 & 31,992 & 21,196 \\
XRaySrc & Evans et al. (2010) & 1,880 & 640 & 1,048 \\
\hline
\end{tabular}

Note. - The third column lists the total number of sources in Stripe 82. The fourth column lists the number of sources that are cross-matched with our Stripe 82 photometric light curve (LC) data set (point sources only). The last column shows the number of sources that have difference image LCs as well. In each catalog, the fraction of sources having difference image LCs is about two-thirds. This corresponds to the area fraction covered by camera columns 2-5 in Stripe 82.

TABLE 2

OVERLAP AMONG CATALOGS

\begin{tabular}{c|c|c|c|c}
\hline \hline Reference Catalog & & & & \\
& SpecQSO & PhotoQSO & VarSrc & XRaySrc \\
Comparison Catalog & & & & \\
\hline SpecQSO & 1.000 & 0.221 & 0.097 & 0.078 \\
PhotoQSO & 0.856 & 1.000 & 0.116 & 0.115 \\
VarSrc & 0.691 & 0.212 & 1.000 & 0.066 \\
XRaySrc & 0.015 & 0.006 & 0.002 & 1.000 \\
\hline
\end{tabular}

Note. - Fractions of sources in one reference catalog that are also found in the comparison catalog.

TABLE 3

Completeness for various $\tau$ CRITERIa estimated for the SPeCQSO SAmple

\begin{tabular}{c|c|c|c}
\hline \hline Selection criterion & $\log (\tau /$ days $) \geq 1.0$ & $\log (\tau /$ days $) \geq 1.5$ & $\log (\tau /$ days $) \geq 2.0$ \\
\hline Bright sample $(i<19)$ & $98.6 \%$ & $96.4 \%$ & $82.2 \%$ \\
Entire sample & $91.4 \%$ & $81.2 \%$ & $56.7 \%$ \\
\hline
\end{tabular}

TABLE 4

COMPlETENESS AND EFFICIENCY FOR VARIOUs AGN SELECTION CRITERIA ESTIMATED For the VARSRC SAMPle

\begin{tabular}{c|c|c}
\hline \hline Selection cuts & Completeness $(\%)$ & Efficiency $(\%)$ \\
\hline $\log (\tau /$ days $) \geq 1.0, \log \left(\hat{\sigma} /\left(\operatorname{counts} / \mathrm{yr}^{1 / 2}\right)\right) \leq 4.15$ & 93.4 & 71.3 \\
$\log (\tau /$ days $) \geq 1.5, \log \left(\hat{\sigma} /\left(\operatorname{counts} / \mathrm{yr}^{1 / 2}\right)\right) \leq 4.15$ & 86.3 & 74.5 \\
$\log (\tau /$ days $) \geq 2.0, \log \left(\hat{\sigma} /\left(\operatorname{counts} / \mathrm{yr}^{1 / 2}\right)\right) \leq 4.15$ & 64.5 & 78.0 \\
\hline
\end{tabular}

